\title{
NBS
}

Eechnical Note

no. 215

\section{PERCENTAGE POINTS OF the BETA DISTRIBUTION}

L. E. VOGLER

U. S. DEPARTMENT OF COMMERCE NATIONAL BUREAU OF STANDARDS 


\section{THE NATIONAL BUREAU OF STANDARDS}

The National Bureau of Standards is a principal focal point in the Federal Government for assuring maximum application of the physical and engineering sciences to the advancement of technology in industry and commerce. Its responsibilities include development and maintenance of the national standards of measurement, and the provisions of means for making measurements consistent with those standards; determination of physical constants and properties of materials; development of methods for testing materials, mechanisms, and structures, and making such tests as may be necessary, particularly for government agencies; cooperation in the establishment of standard practices for incorporation in codes and specifications; advisory service to government agencies on scientific and technical problems; invention and development of devices to serve special needs of the Government; assistance to industry, business, and consumers in the development and acceptance of commercial standards and simplified trade practice recommendations; administration of programs in cooperation with United States business groups and standards organizations for the development of international standards of practice; and maintenance of a clearinghouse for the collection and dissemination of scientific, technical, and engineering information. The scope of the Bureau's activities is suggested in the following listing of its four Institutes and their organizational units.

Institute for Basic Standards. Electricity. Metrology. Heat. Radiation Physics. Mechanics. Applied Mathematics. Atomic Physics. Physical Chemistry. Laboratory Astrophysics." Radio Standards Laboratory: Radio Standards Physics; Radio Standards Engineering. ** Office of Standard Reference Data.

Institute for Materials Research. Analytical Chemistry. Polymers. Metallurgy. Inorganic Materials. Reactor Radiations. Cryogenics. * Office of Standard Reference Materials.

Central Radio Propagation Laboratory.** Ionosphere Research and Propagation. Troposphere and Space Telecommunications. Radio Systems. Upper Atmosphere and Space Physics.

Institute for Applied Technology. Textiles and Apparel Technology Center. Building Research. Industrial Equipment. Information Technology. Performance Test Development. Instrumentation. Transport Systems. Office of Technical Services. Office of Weights and Measures. Office of Engineering Standards. Office of Industrial Services.

* NBS Group, Joint Institute for Laboratory Astrophysics at the University of Colorado.

** Located at Boulder, Colorado. 


\title{
NATIONAL BUREAU OF STANDARDS \\ Eechnical Mote 215 \\ Issued May 30, 1964
}

\section{PERCENTAGE POINTS OF THE BETA DISTRIBUTION}

\author{
L. E. Vogler \\ Central Radio Propagation Laboratory \\ National Bureau of Standards \\ Boulder, Colorado
}

NBS Technical Notes are designed to supplement the Bureau's regular publications program. They provide a means for making available scientific data that are of transient or limited interest. Technical Notes may be listed or referred to in the open literature.

For sale by the Superintendent of Documents, U. S. Government Printing Office

Washington, D.C. 20402

Price: $25 \%$ 

Percentage Points of the Beta Distribution

L. E. Vogler

Tables are presented of percentage points $\mathrm{x}\left(\mathrm{p} \mid v_{2} / 2, v_{1} / 2\right)$ of the Beta distribution. The percentage points are given to six figures correct to within a unit in the last place for probability levels $\mathrm{p}$ :

$$
\mathrm{p}=0.0001,0.001,0.005,0.01,0.025,0.05 \text {, }
$$

$0.1,0.25,0.5$, and for degrees of freedom $v_{2}$ and $v_{1}$ :

$$
\begin{aligned}
v_{2}= & 1(0.1) 2,2.2,2.5(0.5) 5,6(1.0) 10,12, \\
& 15,20,24,30,40,60,120, \\
v_{1}= & 1(1.0) 10,12,15,20,24,30,40,60,120 .
\end{aligned}
$$

A table of the Complete Beta function for the above values of $v_{1}$ and $v_{2}$ is also included.

The present tables of percentage points of the Beta distribution are the outgrowth of a project started at the Central Radio Propagation Laboratory of the National Bureau of Standards with the purpose of extending existing tables of the Fisher-Snedecor F-distribution to cover lower probability levels $\mathrm{p}$ and include non-integer values of the degrees of freedom $v_{1}$ and $v_{2}$. Values of $F$ for finite $v_{1}$ and $v_{2}$ are most easily obtained through its relationship to the Beta distribution.

Thompson's excellent five-figure tables of Beta function percentage points [Thompson, 1941] have been the basic compilation of this statistic since their publication; however, the probability level does not go below 0.005 and no non-integer values of $v_{1}$ or $v_{2}$ are included. In certain situations the Beta distribution with non-integer 
degrees of freedom may be used to approximate an unknown distribution [Pearson and Hartley, 1956]; also in the analysis of variance, some applications require the use of the F-distribution with noninteger values of $v_{2}$ [Box, $\left.1954 \mathrm{a} ; 1954 \mathrm{~b}\right]$.

It was decided to calculate six-figure percentage points for all of the probability levels and most of the $v_{1}^{\prime}$ s and $v_{2}^{\prime} s$ given in the Thompson tables and to add non-integral entries of $v_{2}$ between 1 and 5 and extend the range of $\mathrm{p}$ down to 0.0001 ; the percentage points are tabulated in table II. The six-figure values would assure fivefigure accuracy in the F-distribution and could also serve as a check on the Thompson tables. A list of the discrepancies encountered is given in table I:

TABLE I

\begin{tabular}{lrrll}
$\mathrm{p}$ & $v_{1}$ & $v_{2}$ & Thompson & Table II \\
\hline 0.5 & 4 & 5 & 0.56444 & 0.564446 \\
0.5 & 5 & 4 & 0.43556 & 0.435554 \\
0.5 & 20 & 1 & 0.023052 & 0.0230514 \\
0.25 & 24 & 2 & 0.023689 & 0.0236884 \\
0.1 & 5 & 1 & $0.0^{2} 34818$ & $0.00^{2} 348189$ \\
0.1 & 15 & 4 & 0.064482 & 0.0644585 \\
0.01 & 8 & 1 & 0.0420897 & 0.04208988 \\
0.01 & 10 & 7 & 0.095627 & 0.0956276 \\
0.01 & 20 & 8 & 0.069456 & 0.0694554 \\
0.01 & 40 & 9 & 0.046956 & 0.0469567 \\
0.01 & 60 & 15 & 0.075824 & 0.0758234 \\
0.005 & 7 & 6 & 0.073619 & 0.0736184 \\
0.005 & 9 & 7 & 0.083707 & 0.0837076 \\
0.005 & 12 & 8 & 0.086787 & 0.0867877 \\
0.005 & 120 & 30 & 0.098050 & 0.0980479
\end{tabular}


The Beta distribution is defined through the cumulative distribution function:

$$
\mathrm{p}\left(\mathrm{x} \mid v_{2} / 2, v_{1} / 2\right)=\left[\mathrm{B}\left(v_{2} / 2, v_{1} / 2\right)\right]^{-1} \int_{0}^{\mathrm{x}} \mathrm{u}^{\left(\nu_{2} / 2\right)-1}(1-u){ }^{\left(\nu_{1} / 2\right)-1} \mathrm{du},
$$

where $\mathrm{B}\left(v_{2} / 2, v_{1} / 2\right)$ denotes the Complete Beta function given by

$\mathrm{B}\left(v_{2} / 2, v_{1} / 2\right) \equiv \int_{0}^{1} \mathrm{u}^{\left(\nu_{2} / 2\right)-1}(1-u)^{\left(\nu_{1} / 2\right)-1} \mathrm{du}=\frac{\Gamma\left(\nu_{2} / 2\right) \Gamma\left(\nu_{1} / 2\right)}{\Gamma\left[\left(v_{2} / 2\right)+\left(\nu_{1} / 2\right)\right]}$.

Values of (2) for the $v_{1}^{\prime}$ s and $v_{2}^{\prime}$ 's used in the computations of this paper are shown in table III. Note that $B\left(v_{2} / 2, v_{1} / 2\right)=B\left(v_{1} / 2, v_{2} / 2\right)$ and some of the values in the table are repeated for the convenience of the reader.

The percentage points $\mathrm{x}$ are calculated by using (1) with given values of $p, \nu_{1}$, and $\nu_{2}$. The integral in (1) is expressed in series form and, since $0<x<1$ and $v_{1}$ and $v_{2}$ are finite, rootsolving methods are applied to evaluate $x$. .Unfortunately, no single series representation is computationally practical for all values of $v_{1}$ and $v_{2}$. Consequently, different methods must be used for different ranges of the degrees of freedom.

After considerable experimentation, two expansions of (1) were found that could be used in the area covered by the tables. For $x$ not too near unity, the following relationship was utilized: 


$$
\mathrm{p}\left(v_{2} / 2\right) \mathrm{B}\left(v_{2} / 2, v_{1} / 2\right)=\mathrm{x}^{v_{2} / 2}(1-\mathrm{x})^{\left(\nu_{1} / 2\right)-1}\left[1+\sum_{\mathrm{k}=1}^{\infty} \mathrm{T}_{\mathrm{k}}(\mathrm{x})\right],
$$

where

$$
T_{k}(x)=\left(\frac{v_{1}-2 k}{v_{2}+2 k}\right)\left(\frac{x}{1-x}\right) \quad T_{k-1}(x), \quad T_{0}(x)=1
$$

For $\mathbf{x}$ near unity, an alternate expression was derived:

$$
(1-\mathrm{p})\left(v_{1} / 2\right) \mathrm{B}\left(v_{2} / 2, v_{1} / 2\right)=(1-\mathrm{x})^{v_{1} / 2}\left[1+\left(v_{1} / 2\right) \sum_{\mathrm{k}=1}^{\infty} \mathrm{T}_{\mathrm{k}}^{\prime}(\mathrm{x})\right] \text {, }
$$

where

$$
T_{k}^{\prime}(x)=\left(\frac{v_{1}+2 k-2}{v_{1}+2 k}\right)\left(\frac{v_{2}-2 k}{2 k}\right)(1-x) T_{k-1}^{\prime}(x), \quad T_{0}^{\prime}(x)=-2 / v_{1} .
$$

Using either (3) or (4) (and occasionally both for checking purposes), $x$ was obtained through an iteration process. Newton's method of root-solving being in general the most practical. As a further check, a third expression was derived for those areas of the tables containing the change-over from (3) to (4):

$$
x_{i+1}=x_{i}\left\{1+\left(2 / v_{2}\right) \sum_{k=1}^{\infty}\left(D_{k}-C_{k}\right) x_{i}^{k}\right\}
$$


where

$$
\begin{gathered}
D_{k}=-\left(\frac{\delta_{i}+1-k}{k}\right) D_{k-1}, D_{0}=1, C_{k}=\left\{\frac{v_{2}+2 k+v_{1}-4}{v_{2}+2 k}\right\} C_{k-1}, C_{1}=\frac{v_{1}-2}{v_{2}+2}, \\
\delta_{i}=\left[\left\{\ln \left(p v_{2} B / 2\right)-\left(v_{2} / 2\right) \ln x_{i}\right\} / \ln \left(1-x_{i}\right)\right]-\left(v_{1}-2\right) / 2,
\end{gathered}
$$

and the subscript $i$ denotes the number of iterations.

Equations (3), (4), and (5) were programmed for an electronic computer, at least eight figures being carried in all arithmetic operations. Successive approximations to the true $\mathrm{x}$ were calculated from the right hand members of (3) or (4) until agreement to at least seven figures was reached with the expressions on the left. The final $x$ was then rounded to six figures.

The main portion of the tables was checked (to five figures) by comparis on with the Thompson tables, differences being re-checked through hand calculations (see table I). For values not given in Thompson, plots were made of $\mathrm{x}$ vs $\mathrm{p}$ for a given $v_{1}$ and $v_{2}$ with the hope of at least avoiding gross errors.

Differencing was not used to check the table entries because of the irregular intervals in $v_{2}$. In those regions where cumulative round-off errors were of possible concern, the $x$-values were checked by independent runs of at least two (and sometimes all three) of the generating equations (3), (4), and (5). As a final check most of the entries were re-calculated using double-precision arithmetic in all machine operations. To avoid copying errors, the reproduced table was compared with the original computer print-outs, and it is believed that all entires are correct to within a unit in the last figure. 
The present tables are constructed only up to $\mathrm{p}=0.5$, thus giving the lower percentage points of the Beta distribution. Corresponding upper percentage points are easily obtained by interchanging $v_{1}$ and $v_{2}$ and replacing $\mathrm{p}$ and $\mathrm{x}$ by $(1-\mathrm{p})$ and $(1-\mathrm{x})$ :

$$
\mathrm{p}\left(\mathrm{x} \mid v_{2} / 2, v_{1} / 2\right)=1-\mathrm{p}\left(1-\mathrm{x} \mid v_{1} / 2, v_{2} / 2\right)
$$

Although the Beta distribution may be used indirectly to obtain a number of important statistical distributions, only two of its applications will be mentioned here.

(a) The Fisher-Snedecor variance ratio $F$ with degrees of freedom $v_{1}$ and $v_{2}$ and of probability $p$ is related to $x$ by

$$
F=\left(\frac{v_{2}}{v_{1}}\right)\left(\frac{1-x}{x}\right)
$$

(b) The relationship to the cumulative sum of the individual terms of the binomial distribution is given by

$$
\sum_{k=m}^{n}\left(\begin{array}{l}
n \\
k
\end{array}\right) x^{k}(1-x)^{n-k}=p(x \mid m, n-m+1)
$$

Interpolation within the tables to a high degree of accuracy is probably most easily accomplished through the method of Lagrangian interpolation. Tables of Lagrangian coefficients for harmonic interpolation have been developed by Comrie and Hartley [1941]; a comprehensive discussion of interpolation is given by Hartley [1941] and tables to facilitate the p-wise interpolation may be found in a paper by Salzer [1951]. For practical applications two or three figures will 
often suffice, in which case a first order harmonic interpolation in the $v$-directions may be used. Thus, if for a given $\mathrm{p}: v_{1}^{\prime} \leq v_{1} \leq v_{1}^{\prime \prime}$, $v_{2}^{\prime} \leq v_{2} \leq v_{2}^{\prime \prime}$, and $\mathrm{x}_{1}^{\prime}\left(v_{1}^{\prime}, v_{2}^{\prime}\right), \mathrm{x}_{1}^{\prime \prime}\left(v_{1}^{\prime \prime}, v_{2}^{\prime}\right), \mathrm{x}_{2}^{\prime}\left(v_{1}^{\prime}, v_{2}^{\prime \prime}\right), \mathrm{x}_{2}^{\prime \prime}\left(v_{1}^{\prime \prime}, v_{2}^{\prime \prime}\right)$ denote the nearest tabulated values, then

$$
\mathrm{x}\left(v_{1}, v_{2}\right)=\delta_{2} \mathrm{x}_{1}+\left(1-\delta_{2}\right) \mathrm{x}_{2}
$$

where

$$
x_{1}=\delta_{1} x_{1}^{\prime}+\left(1-\delta_{1}\right) x_{1}^{\prime \prime}, \quad x_{2}=\delta_{1} x_{2}^{\prime}+\left(1-\delta_{1}\right) x_{2}^{\prime \prime}
$$

and

$$
\delta_{1}=\frac{v_{1}^{\prime}\left(v_{1}^{\prime \prime}-v_{1}\right)}{v_{1}\left(v_{1}^{\prime \prime}-v_{1}^{\prime}\right)}, \quad \delta_{2}=\frac{v_{2}^{\prime}\left(v_{2}^{\prime \prime}-v_{2}\right)}{v_{2}\left(v_{2}^{\prime \prime}-v_{2}^{\prime}\right)}
$$

The function $\log \mathrm{p}$ may then be used for linear interpolation in the p-direction.

The author wishes to acknowledge the valuable assistance of Mr. Judd Payne and Mrs. J. E. Herman who did much of the computer programming and compilation of values, and Mrs. Marjorie F. Munyon who carefully typed the many tabulated entries. His thanks also go to K. A. Norton whose foresight initiated the project and whose guidance made possible the completion of these tables. 


\section{REFERENCES}

Box, G. E.P. (1954 a), Some theorems on quadratic forms applied in the study of analysis of variance problems, I. Effect of inequality of variance in the one-way classification, Ann. Math. Stat. 25, 290-302.

Box, G. E.P. (1954b), Some theorems on quadratic forms applied in the study of analysis of variance problems, II. Effects of inequality of variance and of correlation between errors in the two-way classification, Ann。 Math. Stat。 25, 484-498.

Comrie, L. J., and H. O. Hartley (1941-1942), Table of Lagrangian coefficients for harmonic interpolation in certain tables of percentage points, Biometrika 32 , p. 183.

Hartley, H. O. (1941), Methods of interpolation, Biometrika 32, $161-167$.

Pearson, E.S., and H. O. Hartley (1956), Biometrika tables for statisticians, Vol. I, (Cambridge University Press). See section 8.4 .

Salzer, H. E. (1951), Checking and interpolation of functions tabulated at certain irregular logarithmic intervals, J. Res. Natl. Bur. Std. 46 , No. 1, 74-77.

Thompson, C.M. (1941-1942), Tables of percentage points of the incomplete beta-function, Biometrika 32, p. 151 . 
TABLE II

PERCENTAGE POINTS OF THE BETA DISTRIBUTION 


\begin{tabular}{|c|c|c|c|c|c|c|c|c|c|}
\hline$\nu_{2}$ & $p=0.0001$ & $p=0.001$ & $p=0.005$ & $p=0.01$ & $p=0.025$ & $p=0.05$ & $p=0.1$ & $p=0.25$ & $p=0.5$ \\
\hline 1 & $(-8) 2.46740$ & $(-6) 2.46740$ & $(-5) 6.16838$ & $(-4) 2.46720$ & $(-3) 1.54133$ & $(-3) 6.15583$ & $(-2) 2.44717$ & $(-1) 1.46447$ & $(-1) 5$ \\
\hline 1.1 & $(-7) 1.28058$ & $(-6) 8.42537$ & $(-4) 1.57189$ & $(-4) 5.54235$ & $(-3) 2.93014$ & $(-2) 1.03081$ & $(-2) 3.60442$ & $(-1) 1.81222$ & $(-1) 5.42541$ \\
\hline 1.2 & $(-7) 5.03764$ & $|(-5) 2.33825|$ & $(-4) 3.41820$ & $(-3) 1.08496$ & $(-3) 4.99017$ & $7 \mid(-2) 1.57890$ & $(-2) 4.95874$ & $(-1) 2.15558$ & $(-1) 5.79267$ \\
\hline 1.3 & $(-6) 1.60167$ & $7(-5) 5.53386$ & $(-4) 6.58093$ & $(-3) 1.91093$ & $(-3) 7.81055$ & $(-2) 2.25859$ & $(-2) 6.47480$ & $(-1) 2.48844$ & $(-1) 6.11126$ \\
\hline 1.4 & $(-6) 4.30871$ & $(-4) 1.15586$ & $(-3) 1.15160 \mid$ & $(-3) 3.09810$ & $(-2) 1.14422$ & $(-2) 3.06241$ & $(-2) 8.11651$ & $(-1) 2.80714$ & $(-1) 6.38916$ \\
\hline 1.5 & $(-5) 1.01422$ & $(-4) 2.18493$ & $(-3) 1.86721$ & $(-3) 4.70126$ & $(-2) 1.59001$ & $1(-2) 3.97876$ & $(-2) 9.85004$ & $(-1) 3.10975$ & $(-1) 6.63300$ \\
\hline 1.6 & $(-5) 2 \cdot 14216$ & $(-4) 3.80898$ & $(-3) 2.84592$ & $(-3) 6.76140$ & $(-2) 2.11692$ & $f(-2) 4.99376$ & $(-1) 1.16454$ & $(1-1) 3.39547 \mid$ & $(-1) 6.84818$ \\
\hline 1.7 & $(-5) 4.13867$ & $(1-4) 6.21246$ & $(-3) 4.12258$ & $(-3) 9.30489$ & $(-2) 2.72110$ & $(-2) 6.09264$ & $(-1) 1.34772$ & $(-1) 3.66428$ & $(-1) 7.03915$ \\
\hline 1.8 & $(-5) 7.42418$ & $(-4) 9.58647$ & $(-3) 5.72461$ & $(-2) 1.23442$ & $(-2) 3.39705$ & $(-2) 7.26073$ & $(-1) 1.53243$ & $(-1) 3.91663$ & $(-1) 7.20954$ \\
\hline 1.9 & $(-4) 1.25122$ & $(-3) 1.41196$ & $(-3) 7.67150$ & $(-2) 1.58793$ & $(-2) 4.13822$ & $(-2) 8.48411$ & $(-1) 1.71697$ & $(-1) 4.15325$ & $(-1) 7.36234$ \\
\hline 2 & $(-4) 1.99990$ & $(-3) 1.99900$ & $(-3) 9.97500$ & $(-2) 1.99000$ & $(-2) 4.93750$ & $(-2) 9.75000$ & $(-1) 1.90000$ & $(-1) 4.37500$ & $(-1) 7$ \\
\hline 2.2 & $(-4) 4.48703$ & $(-3) 3.63681$ & $(-2) 1.56637$ & $(-2) 2.93172$ & $(-2) 6.68131$ & $(-1) 1.23648$ & $(-1) 2.25763$ & $(-1) 4.77771$ & $(-1) 7.73779$ \\
\hline 2.5 & $(-3) 1.17884$ & $(-3) 7.42762$ & $(-2) 2.67998$ & $(-2) 4.64520$ & $(-2) 9.55746$ & $(-1) 1.63632$ & $(-1) 2.76412$ & $(-1) 5.29365$ & $(-1) 8.02247$ \\
\hline 3 & $(-3) 3.81191$ & $(-2) 1.76440$ & $(-2) 5.12365$ & $(-2) 8.08272$ & $(-1) 1.46746$ & $(-1) 2.28520$ & $(-1) 3.51357$ & $(-1) 5.97150$ & $(-1) 8.36806$ \\
\hline 3.5 & $(-3) 8.76150$ & $(-2) 3.25162$ & $(-2) 8.08201$ & $(-1) 1.19192$ & $(-1) 1.97938$ & $3(-1) 2.88205$ & $(-1) 4.14731$ & $(-1) 6.48611$ & $(-1) 8.61240$ \\
\hline 4 & $(-2) 1.62854$ & $(-2) 5.11919$ & $(-1) 1.13209$ & $(-1) 1.58745$ & $(-1) 2.46642$ & $(-1) 3.41628$ & $(-1) 4.68123$ & $(-1) 6.88776$ & $(-1) 8.79385$ \\
\hline 4.5 & $(-2) 2.62922$ & $(-2) 7.26186$ & $(-1) 1.46647$ & $(-1) 1.97741$ & $(-1) 2.91813$ & $(-1) 3.88943$ & $(-1) 5.13312$ & $(-1) 7.20892$ & $(-1) 8.93372$ \\
\hline 5 & $(-2) 3.84789$ & $(-2) 9.58207$ & $(-1) 1.79955$ & $(-1) 2.35204$ & $(-1) 3.33178$ & $(-1) 4.30741$ & $(-1) 5.51851$ & $(-1) 7.47108$ & 04474 \\
\hline 6 & $(-2) 6.78050$ & $(-1) 1.44552$ & $(-1) 2.43556$ & $(-1) 3.03874$ & $(-1) 4.05047$ & $(-1) 5.00526$ & $(-1) 6.13751$ & $(-1) 7.87255$ & 20967 \\
\hline 7 & $(-1) 1.01205$ & $(-1) 1.93129$ & $(-1) 3.01262$ & $(-1) 3.63705$ & $(-1) 4.64417$ & $(-1) 5.55933$ & $(-1) 6.61037$ & $(-1) 8.16496$ & $(-1) 9.32622$ \\
\hline 8 & $(-1) 1.36299$ & $(-1) 2.39415$ & $(-1) 3.52606$ & $(-1) 4.15398$ & $(-1) 5.13779$ & $(-1) 6.00706$ & $(-1) 6.98207$ & $(-1) 8.38716$ & $(-1) 9.41289$ \\
\hline 9 & $(-1) 1.71505$ & $(-1) 2.82511$ & $(-1) 3.97990$ & $(-1) 4.60089$ & $(-1) 5.55237$ & $(-1) 6.37513$ & $(-1) 7.28137$ & $(-1) 8.56159$ & $(-1) 9.47985$ \\
\hline 10 & $(-1) 2.05858$ & $(-1) 3.22169$ & $(-1) 4.38088$ & $(-1) 4.98895$ & $(-1) 5.90433$ & $(-1) 6.68244$ & $(-1) 7.52728$ & $(-1) 8.70211$ & $(-1) 9.53313$ \\
\hline 12 & $(-1) 2.70106$ & $(-1) 3.91602$ & $(-1) 5.05173$ & $(-1) 5.62582$ & $(-1) 6.46769$ & $(-1) 7.16537$ & $(-1) 7.90694$ & $(-1) 8.91443$ & $(-1)$ \\
\hline 15 & $(-1) 3.53373$ & $(-1) 4.74873$ & $(-1) 5.81439$ & $(-1) 6.33363$ & $(-1) 7.07564$ & $(-1) 7.67535$ & $(-1) 8.29959$ & $(-1) 9.12849$ & $(-1)$ \\
\hline 20 & $(-1) 4.60835$ & $(-1) 5.74403$ & $(-1) 6.67915$ & $(-1) 7.11846$ & $(-1) 7.73052$ & $(-1) 8.21313$ & $(-1) 8.70525$ & $(-1) 9.34420$ & $(-1) 9.76949$ \\
\hline 24 & $(-1) 5.25587$ & $(-1) 6.31114$ & $(-1) 7.15323$ & $(-1) 7.54175$ & $(-1) 8.07628$ & $(-1) 8.49267$ & $(-1) 8.91295$ & $(-1) 9.45263$ & $(-1) 9.80832$ \\
\hline 30 & $(-1) 5.98897$ & $(-1) 6.92952$ & $(-1) 7.65703$ & $(-1) 7.98669$ & $(-1) 8.43466$ & $(-1) 8.77941$ & $(-1) 9.12389$ & $(-1) 9.56142$ & $(-1) 9$ \\
\hline 40 & $(-1) 6.81797$ & $(-1) 7.60321$ & $(-1) 8.19204$ & $(-1) 8.45414$ & $(-1) 8.80593$ & $(-1) 9.07343$ & $(-1) 9.33808$ & $(-1) 9.67057$ & $(-1) c$ \\
\hline 60 & $(-1) 7.75425$ & $(-1) 8.33646$ & $(-1) 8.75981)$ & $(-1) 8.94493$ & $(-1) 9.19039$ & $(-1) 9.37483$ & $(-1) 9.55550$ & $(-1) 9.78006$ & $(-1) 9.92383$ \\
\hline 120 & $(-1) 8.81033$ & $(-1) 9.13380$ & $(-1) 9.36192$ & $(-1) 9.45993$ & $(-1) 9.58832$ & $(-1) 9.68366$ & $(-1) 9.77614$ & $(-1) 9.88988$ & $(-1) 9.96200$ \\
\hline$\infty$ & 1.00000 & 1.00000 & 1.00000 & 1.00000 & 1.00000 & 1.00000 & 1.00000 & 1.00000 & 1.00000 \\
\hline
\end{tabular}


$\nu_{1}=2$

\begin{tabular}{|c|c|c|c|c|c|c|c|c|c|}
\hline$\nu_{2}$ & $p=0.0001$ & $p=0.001$ & $p=0.005$ & $p=0.01$ & $p=0.025$ & $p=0.05$ & $p=0.1$ & $p=0.25$ & $p=0.5$ \\
\hline I & $(-8) 1.00000$ & $(-6)$ & $(-5) 2.50000$ & $(-4) 1.00000$ & $(-4) 6.25000$ & $(-3) 2.50000$ & $(-2) 1.00000$ & $(-2) 6.25000$ & $(-1) 2.50000$ \\
\hline 1.1 & $(-8) 5.33670)$ & $(-6) 3.51119$ & $(-5) 6.55102$ & $(-4) 2.31013$ & $(-3) 1.22226$ & $(-3) 4.31014$ & $(-2) 1.51991$ & $(-2) 8.04166$ & $(-1) 2.83578$ \\
\hline 1.2 & $(-7) 2.15443 \mid$ & $(-5) 1.00000$ & $(-4) 1.46201$ & $(-4) 4.64159$ & $(-3) 2.13747$ & $(1-3) 6.78604$ & $(-2) 2.15443$ & $(-2) 9.92126$ & $(-1) 3.14980$ \\
\hline 1.3 & $(-7) 7.01704$ & $(-5) 2.42446$ & $(-4) 2.88372$ & $(-4) 8.37678$ & $(-3) 3.42999$ & $(-3) 9.96358$ & $(-2) 2.89427$ & $(-1) 1.18510$ & $(-1) 3.44252$ \\
\hline 1.4 & $(-6) 1.93070$ & $(-5) 5.17947$ & $(-4) 5.16196$ & $(-3) 1.38950$ & $(-3) 5.14450$ & $(-2) 1.38480$ & $(-2)$ & $(-1) 1.38011$ & 499 \\
\hline 1.5 & $(-6) 4.64159$ & $(-4) 1.00000$ & $(-4) 8.54988$ & $(-3) 2.15443$ & $(-3) 7.31004$ & $(-2) 1.84202$ & $(-2) 4.64159$ & $(-1) 1.57490$ & 6850 \\
\hline 1.6 & $(-5) 1.00000$ & $(-4) 1.77828$ & $(-3) 1.32957$ & $(-3) 3.16228$ & $(-3) 9.94088$ & $(-2) 2.36435$ & $(-2) 5.62341$ & $(-1) 1.76777$ & $(-1)$ \\
\hline 1.7 & $(-5) 1.96842$ & $(-4) 2.95521$ & $(-3) 1.96293$ & $(-3) 4.43669$ & $(-2) 1.30384$ & $(-2) 2.94698$ & $(-2) 6.66085$ & $(-1) 1.95747$ & 2433 \\
\hline 1.8 & $(-5) 3.59381$ & $(-4) 4.64159$ & $(-3) 2.77524$ & $(-3) 5.99484$ & $(-2) 1.65933$ & $(-2) 3.58436$ & $(-2) 7$ & $(-1)^{2}$ & $(-1)$ \\
\hline 1.9 & $(-5) 6.15848$ & $(-4) 6.95193$ & $(-3) 3.78323$ & 84760 & $(-2) 2.05883$ & $(-2) 4$ & $(-2)$ & 409 & 088 \\
\hline 2 & $(-4) 1.00000$ & $(-3) 1.00000$ & $(-3) 5.00000$ & $(-2) 1.00000$ & $(-2) 2.50000$ & $(-2) 5.00000$ & $(-1) 1.00000$ & $(-1) 2.50000$ & 00000 \\
\hline 2.2 & $(-4) 2.31013$ & $(-3) 1.87382$ & $(-3) 8.09384$ & $(-2) 1.51991$ & $(-2) 3.49608$ & $(-2) 6.56516$ & $(-1) 1.23285$ & $(-1) 2.83578$ & $(-1) 5$ \\
\hline 2.5 & $(-4) 6.30957$ & $(-3) 3.98107$ & $(-2) 1.44270$ & $(-2) 2.51189$ & $(-2) 5.22820$ & $(-2) 9.10282$ & 58489 & $(-1) 3.29877$ & $(-1) 5.74349$ \\
\hline 3 & $(-3) 2$ & $(-2) 1$ & $(-2) 2.92402$ & $(-2) 4.64159$ & $(-2) 8.54988$ & $(-1) 1.35721$ & $(-1) 2.15443$ & $(-1) 3.96850$ & $(-1) 6.29961$ \\
\hline 3.5 & $(-3) 5.17947$ & $(-2) 1.93070$ & $(-2) 4$ & $(-2) 7.19686$ & $(-1) 1.21489$ & $(-1) 1$ & $(-1)$ & 862 & 950 \\
\hline 4 & $(-2) 1.00000$ & $(-2) 3.16228$ & $(-2) 7.07107$ & $(-1) 1.00000$ & $(-1) 1.58114$ & $=(-1) 2.23607$ & $(-1) 3.16228$ & $3(-1) 5.00000$ & $(-1)$ \\
\hline 4.5 & $(-2) 1.66810$ & $(-2) 4.64159$ & $(-2) 9.49118$ & $(-1) 1.29155$ & $(-1) 1.94077$ & $(-1) 2.64098$ & $(-1) 3.59381$ & $(-1) 5.40030$ & $(-1) 7.34867$ \\
\hline 5 & $(-2) 2.51189$ & $(-2) 6.30957$ & $(-1) 1.20112$ & $(-1) 1.58489$ & $(-1) 2.28653$ & $(-1) 3.01709$ & $(-1) 3.98107$ & $7(-1) 5.74349$ & $(-1)$ \\
\hline 6 & $(-2) 4$ & $(-1) 1$ & $(-1) 1$ & $(-1) 2$ & 92402 & $(-1) 3$ & $(-1) 4$ & $9 \mid(-1) 6.29961$ & $(-1) 7$ \\
\hline 7 & $(-2) 7.19686$ & $(-1) 1.38950$ & $(-1) 2.20071$ & $(-1) 2.68270$ & $(-1) 3.48553$ & $(-1) 4.24891$ & $(-1) 5.17947$ & $(-1) 6.72950$ & $(-1) 8$ \\
\hline 8 & $(-1)$ & $(-1)$ & 915 & $(-1)$ & $(-1)$ & 2871 & $(-1) 5$ & $1(-1) 7.07107$ & $(-1) 8.40896$ \\
\hline 9 & $(-1) 1.29155$ & $(-1) 2.15443$ & $(-1) 3.08078$ & $(-1) 3.59381$ & $(-1) 4.40541$ & $(-1) 5.13904$ & $(-1) 5.99484$ & $(-1) 7.34867$ & $(-1) 8.57244$ \\
\hline 10 & $(-1) 1$. & $(-1)^{2}$ & $(-1) 3.46572$ & $(-1) 3.98107$ & $(-1) 4.78176$ & $(-1) 5.49280$ & $(-1) 6.30957$ & $(-1) 7.57858$ & $(-1) 8.70551$ \\
\hline 12 & $(-1) 2.15443$ & $(-1) 3$ & $(-1) 4.13519$ & $(-1) 4.64159$ & $(-1) 5.40742$ & $(-1) 6.06962$ & $(-1) 6.81292$ & $(-1) 7.93701$ & $(-1) 8.90899$ \\
\hline 15 & $(-1) 2.92864$ & $(-1) 3.98107$ & $(-1) 4.93396$ & $(-1) 5.41170$ & $(-1) 6.11494$ & $=(-1) 6.70702$ & $(-1) 7.35642$ & $(-1) 8.31238$ & $(-1) 9.11722$ \\
\hline 20 & $(-1) 3.98107$ & $(-1) 5.01187$ & $(-1) 5$ & $(-1) 6.30957$ & $(-1) 6.91503$ & $(-1) 7.41134$ & $(-1) 7.94328$ & $(-1) 8.70551$ & $(-1) 9.33033$ \\
\hline 24 & $(-1) 4$. & 2341 & $(-1) 6.43054$ & $(-1) 6.81292$ & $(-1) 7.35352$ & $(-1) 7.79078$ & $(-1) 8.25404$ & $(-1) 8.90899$ & $(-1) 9.43874$ \\
\hline 30 & $(-1) 5$ & $(-1) 6.30957$ & 2422 & $(-1) 7.35642$ & $(-1) 7.81981$ & $(-1) 8.18964$ & $(-1) 8.57696$ & $(-1) 9.11722$ & $(-1) 9.54842$ \\
\hline 40 & 957 & $(-1)$ & $(-1) 7$ & $(-1) 7.94328$ & $(-1) 8.31567$ & $(-1) 8.60892$ & $(-1) 8.91251$ & $(-1) 9.33033$ & $(-1) 9.65936$ \\
\hline 60 & $(-1) 7.35642$ & $(-1) 7.94328$ & $(-1) 8.38106$ & $(-1) 8.57696$ & $(-1) 8.84297$ & $(-1) 9.04966$ & $(-1) 9.26119$ & $(-1) 9.54842$ & $(-1) 9.77160$ \\
\hline 120 & $(-1) 8.57696$ & $(-1) 8$ & $(-1) 9$ & $(-1) 9$ & 0371 & $(-1) 9.51297$ & $(-1) 9.62351$ & $(-1) 9.77160$ & $(-1) 9.88514$ \\
\hline$\infty$ & 1.00000 & 1.00000 & 1.00000 & 1.00000 & 1.00000 & 1.00000 & 1.00000 & 1.00000 & 1.00000 \\
\hline
\end{tabular}


$\nu_{1}=3$

\begin{tabular}{l|c|c|c|c|c|c|c|c|c}
\hline$\nu_{2}$ & $p=0.0001$ & $p=0.001$ & $p=0.005$ & $p=0.01$ & $p=0.025$ & $p=0.05$ & $p=0.1$ & $p=0.25$ & $p=0.5$ \\
\hline \hline
\end{tabular}

\begin{tabular}{l|l|l|l|l|l|l|l|l|}
$(-9) 6.16850$ & $(-7) 6.16850$ & $(-5) 1.54213$ & $(-5) 6.16863$ & $(-4) 3.85581$ & $(-3) 1.54292$ & $(-3) 6.18124$ & $(-2) 3.90631$ & $(-1) 1.63194$
\end{tabular} $1.1(-8) 3.32319(-6) 2.18644(-5) 4.07941)(-4) 1.43860(-4) 7.61294(-3) 2.68627(-3) 9.49366(-2) 5.09155)(-1) 1.88195$ $1.2(-7) 1.35370(-6) 6.28333(-5) 9.18654(-4) 2.91673(-3) 1.34361(-3) 4.26959(-2) 1.35948)(-2) 6.36104(-1) 2.12294$ $1.3(-7) 4.44703(-5) 1.53650(-4) 1.82765(-4) 5.30961)(-3) 2.17518(-3) 6.32652(-2) 1.84456)(-2) 7.69123(-1) 2.35408$ $1.4(-6) 1.23364(-5) 3.30952)(-4) 3.29861)(-4) 8.88067(-3) 3.29032)(-3) 8.87149(-2) 2.39876)(-2) 9.06251)(-1) 2.57508$ $1.5(-6) 2.98914)(-5) 6.44001(-4) 5.50690(-3) 1.38798(-3) 4.71394(-2) 1.19029(-2) 3.01521(-1) 1.04589(-1) 2.78597$ $1.6(-6) 6.48841(-4) 1.15386(-4) 8.62887(-3) 2.05298(-3) 6.46165(-2) 1.54069(-2) 3.68664 \mid(-1) 1.18677)(-1) 2.98699)$ $1.7(-5) 1.28641(-4) 1.93140(-3) 1.28327(-3) 2.90176 \mid(-3) 8.54063(-2) 1.93608(-2) 4.40582)(-1) 1.32785(-1) 3.17850$ $1.8(-5) 2.36493(-4) 3.05465(-3) 1.82713(-3) 3.94902(-2) 1.09509(-2) 2.37357(-2) 5.16583(-1) 1.46834(-1) 3.36093$ $1.9(-5) 4.07960(-4) 4.60570(-3) 2.50774)(-3) 5.20543(-2) 1.36864(-2) 2.84993(-2) 5.96021)(-1) 1.60761)(-1) 3.53474$ $2 \quad(-5) 6.66678(-4) 6.66778(-3) 3.33612(-3) 6.67783(-2) 1.67369(-2) 3.36175(-2) 6.78302)(-1) 1.74518(-1) 3.70039$ $2.2(-4) 1.55881(-3) 1.26474(-3) 5.46843(-2) 1.02808(-2) 2.37244(-2) 4.47797(-2) 8.49310)(-1) 2.01383(-1) 4.00905$ $2.5(-4) 4.32869(-3) 2.73261)(-3) 9.91860(-2) 1.72979(-2) 3.61573(-2) 6.33473(-1) 1.11551)(-1) 2.39744(-1) 4.42179$ $3(-3) 1.51437(-3) 7.03688(-2) 2.06325(-2) 3.28335(-2) 6.08303(-2) 9.73082(-1) 1.56476)(-1) 2.98014(-1) 5.00000$ $3.5(-3) 3.71794(-2) 1.38848(-2) 3.49658(-2) 5.21263(-2) 8.86091)(-1) 1.32823(-1) 2.00123)(-1) 3.49355(-1) 5.47196)$ $4 \quad(-3) 7.31190(-2) 2.31842(-2) 5.20987(-2) 7.39602)(-1) 1.17863(-1) 1.68250(-1) 2.41363 \mid(-1) 3.94477(-1) 5.86373$ $4.5(-2) 1.23989(-2) 3.46210(-2) 7.12092(-2) 9.73164(-1) 1.47479)(-1) 2.02652(-1) 2.79773(-1) 4.34217(-1) 6.19379$ $5(-2) 1.89484(-2) 4.77978(-2) 9.15930(-1) 1.21419(-1) 1.76736(-1) 2.35534(-1) 3.15290(-1) 4.69360(-1) 6.47548$ $6 \quad(-2) 3.59193(-2) 7.78110(-1) 1.34079(-1) 1.69786(-1) 2.32591(-1) 2.95987(-1) 3.78160(-1) 5.28481)(-1) 6.93053$ $7 \quad(-2) 5.68970(-1) 1.10555(-1) 1.76557(-1) 2.16358(-1) 2.83752)(-1) 3.49293(-1) 4.31507(-1) 5.76094)(-1) 7.28193$ $8(-2) 8.05085(-1) 1.44174(-1) 2.17454(-1) 2.59968(-1) 3.29926(-1) 3.96068(-1) 4.77003(-1) 6.15157(-1) 7.56136$ $9(-1) 1.05620(-1) 1.77496(-1) 2.56037(-1) 3.00243(-1) 3.71374(-1) 4.37155(-1) 5.16097(-1) 6.47732)(-1) 7.78880$ $10 \quad(-1) 1.31384(-1) 2.09827(-1) 2.92037(-1) 3.37189(-1) 4.08549(-1) 4.73377(-1) 5.49964(-1) 6.75286)(-1) 7.97751$

12

15

20

24

30

40

60

120 $(-1) 1.82653(-1) 2.70194(-1) 3.56323(-1) 4.01907(-1) 4.72022(-1) 5.34024(-1) 6.05554(-1) 7.19307(-1) 8.27249$ $(-1) 2.54647(-1) 3.48790(-1) 4.3569 q(-1) 4.79986(-1) 5.46281(-1) 6.03327(-1) 6.67576)(-1) 7.66843)(-1) 8.58268$ $(-1) 3.56193(-1) 4.51517(-1) 5.34001(-1) 5.74472(-1) 6.33391(-1) 6.82706(-1) 7.36913(-1) 8.18249(-1) 8.90923$ $4(-1) 4.21858(-1) 5.14322(-1) 5.91760(-1) 6.29027(-1) 6.82531(-1) 7.26692(-1) 7.74639(-1) 8.45528(-1) 9.07901$ $(-1) 5.00145(-1) 5.86357(-1) 6.56220(-1) 6.89192(-1) 7.35870(-1) 7.73857(-1) 8.14593(-1) 8.73930(-1) 9.25336$ $(-1) 5.93619(-1) 6.69080(-1) 7.28232(-1) 7.55605(-1) 7.93812)(-1) 8.24466(-1) 8.56930(-1) 9.03514(-1) 9.43244$ $(-1) 7.05402(-1) 7.64198(-1) 8.08767)(-1) 8.28985(-1) 8.56806(-1) 8.78809(-1) 9.01819(-1) 9.34341)(-1) 9.61645$ $20(-1) 8.39307(-1) 8.73718(-1) 8.98934(-1) 9.10144(-1) 9.25350)(-1) 9.37203(-1) 9.49442(-1) 9.66480(-1) 9.80556$ $\infty$ $1.00000 \quad 1.00000 \quad 1.00000$ $1.00000 \quad 1.00000$ 1.00000 1.00000 1.00000 1.00000 


\begin{tabular}{|c|c|c|c|c|c|c|c|c|c|}
\hline$\nu_{2}$ & $p=0.0001$ & $p=0.001$ & $p=0.005$ & $p=0.01$ & $p=0.025$ & $p=0.05$ & $p=0.1$ & $p=0.25$ & $p=0.5$ \\
\hline 1 & 444 & $(-7) 4.44445$ & $(-5) 1.11112$ & $(-5) 4.44458$ & $8(-4) 2.77829$ & $(-3) 1.11194$ & $(-3) 4.45768$ & 3095 & 0615 \\
\hline I.I & $(-8) 2$ & $(-6) 1.58270$ & $(-5) 2.95297$ & $(-4) 1.04138$ & g) $(-4) 5.51138$ & $(-3) 1.94526$ & $(-3) 6.88163$ & $(-2)^{3}$ & 0257 \\
\hline 1.2 & 4313 & $(-6) 4.56879$ & $(-5) 6.67987$ & $(-4) 2.12092$ & $2(-4) 9.77159$ & $(-3) 3.10642$ & $(-3) c$ & $7(-?)$ & $(-1)$ \\
\hline 1.3 & $(-7) 3.24761$ & $(-5) 1.12209$ & $(-4) 1.33474$ & $(-4) 3.87783$ & $3(-3) 1.58899$ & $(-3) 4.62427$ & $7(-2) 1$ & 67912 & $(-1) 1.78205$ \\
\hline 1.4 & 4694 & $(-5) 2.42705$ & $(-4) 2.41915$ & $(-4) 6.51345$ & $5(-3) 2.41405$ & $(-3) 6.51387$ & $(-2) 1$ & $(-2) 6.73197$ & 96360 \\
\hline 1.5 & $(-6)$ & $(-5) 4$ & $(-4) 4.05518$ & $3(-3) 1.02220$ & $0(-3) 3.47322$ & $(-3) 8.77860$ & $(-2) 2.22933$ & $(-2) 7.81500$ & $(-1) 2.13923$ \\
\hline 1.6 & $(-6) 4$ & $(-5) 8$ & $(-4) 6.37934$ & $(-3) 1.51801$ & $1(-3) 4.78067$ & $(-2) 1.14125$ & $(-2) 2.73878$ & 91847 & 30887 \\
\hline 1.7 & 4553 & $(-4) 1$ & 52379 & $(-3) 2.15400$ & $0(-3) 6.34447$ & $(-2) 1.44029$ & $(-2) 3.28843$ & 00344 & 47253 \\
\hline 1.8 & $(-5)$ & $(-4)$ & $(-3) 1$ & $(-3)^{2}$ & 16728 & 77319 & $(-2) 3$ & $(-1)$ & 3031 \\
\hline 1.9 & 5) 3.04916 & $(-4) 3.44257$ & $(-3) 1.87491$ & $(-3) 3.89319$ & 02473 & $(-2) 2.13787$ & $(-2) 4.48929$ & $(-1) 1.22787$ & 8239 \\
\hline 2 & $(-5) 5$ & $(-4) 5.00125$ & $(-3) 2.50313$ & $(-3) 5.01256$ & $6(-2) 1.25791$ & $(-2) 2.53206$ & $(-2) 5.13167$ & $(-1) 1.33975$ & $(-1) 2.92893$ \\
\hline 2.2 & $(-4) 1.17689$ & $(-4) 9.54989$ & $(-3) 4.13127$ & $(-3) 7.77145$ & $5(-2) 1.79634$ & $(-2) 3.39948$ & $(-2) 6.48064$ & $(-1) 1.56109$ & $(-1) 3.20627$ \\
\hline 2.5 & $(-4)$ & $(-3) 2$ & $(-3) 7.56647$ & $(-2) 1.32073$ & $3(-2) 2.76687$ & $(-2) 4.86347$ & $(-2) 8.61580$ & $(-1) 1.88370$ & $(-1) 3.58610$ \\
\hline 3 & $(-3) 1.17015$ & $(-3) 5.44068$ & $(-2) 1.59763$ & $(-2) 2.54583$ & $3(-2) 4.73157$ & $(-2) 7.60095$ & $(-1) 1.23099$ & $(-1) 2.38845$ & $(-1) 4.13627$ \\
\hline 3.5 & $(-3) 2.90870$ & $(-2) 1.08740$ & $(-2) 2.74442$ & $(-2) 4.09878$ & $8(-2) 6.99504$ & $(-1) 1.05374$ & $(-1)$ & $(-1) 2.84802$ & 0178 \\
\hline 4 & $(-3) 5$ & $(-2) 1.83703$ & $(-2) 4.14002$ & $(-2) 5.89031$ & $1(-2) 9$ & $(-1) 1.35350$ & $(-1) 1.95800$ & $(-1) 3.26352$ & $(-1) 5.00000$ \\
\hline 4.5 & $(-3) 9.90940$ & $(-2) 2.77271$ & $(-2) 5.72294$ & $(-2) 7.84117$ & $(-1) 1.19434$ & $(-1) 1.65077$ & $(-1) 2.29885$ & $(-1) 3.63859$ & $(-1) 5.34419$ \\
\hline 5 & $(-2) 1.52855$ & $(-2) 3.86578$ & $(-2) 7.43779$ & $(-2) 9.88773$ & $3(-1) 1.44711$ & $(-1) 1.94034$ & $(-1) 2.62044$ & $(-1) 3.97749$ & $(-1) 5.64446$ \\
\hline 6 & $(-2) 2.94587$ & $(-2) 6.40381$ & $(-1) 1.10885$ & $(-1) 1.40868$ & $3(-1) 1.94120$ & $(-1) 2.48605$ & $(-1)^{3}$ & $(-1)$ & 4272 \\
\hline 7 & $(-2) 4.73331$ & $(-2) 9.23581$ & $(-1) 1.48299$ & $(-1) 1.82355$ & $5(-1) 2.40629$ & $(-1) 2.98110$ & $(-1) 3.71508$ & $(-1) 5.04943$ & $(-1)$ \\
\hline 8 & $(-2) 6.78131$ & $(-1) 1.22014$ & $=(-1) 1.85097$ & $(-1) 2.22072$ & $(-1) 2.83582$ & $(-1) 3.42592$ & $(-1) 4.16110$ & $(-1) 5.45819$ & $(-1) 6.86190$ \\
\hline 9 & $(-2) 8.99421$ & $(-1) 1.51921$ & $(-1) 2.20461$ & $(-1) 2.59453$ & $3(-1) 3.22901$ & $(-1) 3.82452$ & $(-1) 4.55219$ & $(-1) 5.80601$ & $(-1) 7.12972$ \\
\hline 10 & $(-1) 1.12969$ & $(-1) 1$ & $(-1)$ & $(-1) 2.94314$ & $4(-1) 3$ & $(-1) 4.18197$ & $(-1) 4.89684$ & $(-1) 6.10521)$ & 5550 \\
\hline 12 & $(-1) 1.59637$ & $(-1) 2.37478$ & $(-1) 3.15088$ & $(-1) 3.56635$ & $5(-1) 4.21277$ & $(-1) 4.79297$ & $(-1) 5.47435$ & $(-1) 6.59290$ & 1510 \\
\hline 15 & $(-1) 2.26817$ & $(-1) 3.12430$ & $(-1) 3.92551$ & $(-1) 4.33867$ & $(-1) 4.96408$ & $(-1) 5.51019$ & $(-1) 6.13604$ & $(-1) 7.13315$ & 232 \\
\hline 20 & $(-1) 3.24365$ & $(-1)$ & $(-1) 4.91435$ & $(-1) 5.30184$ & $(-1) 5.87220$ & $(-1) 6.35641$ & $(-1) 6.89757$ & $(-1) 7.73373$ & $(-1) 8.52037$ \\
\hline 24 & & $(-1) 4$ & $(-1) 5.50975$ & 173 & $3(-1) 6$. & $(-1) 6$ & $(-1) 7.32164$ & $(-1) 8.05946$ & $(-1) 8.74209$ \\
\hline 30 & 73 & $1-1$ & $(-1)$ & 162 & 679 & 6043 & $(-1) 7.77828$ & $(-1) 8.40389$ & $(-1) 8.9$ \\
\hline 40 & $(-1) 5.63088$ & $(-1) 6.37018$ & $(-1) 6.95711$ & $(-1) 7.23155$ & $(-1) 7.61840$ & $(-1) 7.93275$ & $(-1) 8.27065$ & $(-1) 8.76847$ & $(-1) 9.21356$ \\
\hline 60 & $-1) 6.7$ & $(-1) 7.38672$ & $(-1) 7.83703$ & 180 & $(-1) 8.32979$ & $(-1) 8.55910$ & $(-1) 8.80231$ & 15481 & $(-1) 9$. \\
\hline 120 & 3367 & $(-1) 8.58439$ & $(-1)$ & $(-1) 8.96070$ & $(-1) 9.12012$ & $(-1) 9.24578$ & $(-1) 9.37726$ & $(-1) 9.56467$ & $(-1) 9.72639$ \\
\hline$\infty$ & 1.00000 & 1.00000 & 1.00000 & 1.00000 & 1.00000 & 1.00000 & 1.00000 & 1.00000 & 1.00000 \\
\hline
\end{tabular}




\begin{tabular}{|c|c|c|c|c|c|c|c|c|c|}
\hline$\nu_{2}$ & $p=0.0001$ & $p=0.001$ & $p=0.005$ & $p=0.01$ & $p=0.025$ & $p=0.05$ & $p=0.1$ & $p=0.25$ & $p=0.5$ \\
\hline 1 & $(-9) 3.46978$ & $(-7) 3.46978$ & $(-6) 8.67453$ & $(-5) 3.4699 d$ & $(-4) 2.16908$ & $3(-4) 8.68199$ & $9(-3) 3.48189$ & $9(-2) 2.21735$ & $(-2) 9.55258$ \\
\hline 1.1 & $(-8) 1.88320$ & $(-6) 1.23902$ & $(-5) 2.31175$ & $(-5) 8.15255$ & $(-4) 4.31487$ & $(-3) 1.52319$ & $(-3) 5.39149$ & $(-2) 2.91930$ & $(-1) 1.11637$ \\
\hline 1.2 & $(-8) 7.72638$ & $(-6) 3.58628$ & $(-5) 5.24341$ & $(-4) 1.66486$ & $(-4) 7.67105$ & $(-3)^{2} .43923$ & $(-3) 7.78302$ & $(-2) 3.68364$ & $(-1) 1.27557$ \\
\hline 1.3 & $(-7) 2.55585$ & $(-6) 8.83081$ & $(-4) 1.05045$ & $(-4) 3.05196$ & $(-3) 1.25074$ & $(-3) 3.64112$ & $(-2) 1.06446$ & $(-2) 4.49786$ & $(-1) 1.43196$ \\
\hline 1.4 & $(-7) 7.13794$ & $(-5) 1.91492$ & $(-4) 1.90874$ & $(-4) 5.13940$ & $(-3) 1.90516$ & $(-3) 5.14300$ & $(-2) 1.39523$ & $(-2) 5.35113$ & 58498 \\
\hline 1.5 & $(-6) 1.74083$ & $(-5) 3.75063$ & $(-4) 3.20752$ & $(-4) 8.08583$ & $(-3) 2.74811$ & $(-3) 6.94982$ & $(-2) 1.76748$ & $(-2) 6.23429$ & $(-1) 1$ \\
\hline 1.6 & $(-6) 3.80271$ & $(-5) 6.76264$ & $(-4) 5.05810$ & $(-3) 1.20373$ & $(-3) 3.79219$ & $(-3) 9.05916$ & $(-2) 2.17772$ & $(-2) 7.13965$ & $(-1) 1.87969$ \\
\hline 1.7 & $(-6) 7.58573$ & $(-4) 1.13895$ & $(-4) 7.56920$ & $(-3) 1.71214$ & $(-3) 5.04521$ & $(-2) 1.14630$ & $(-2) 2.62232$ & $(-2) 8.06079$ & $(-1) 2.02110$ \\
\hline 1.8 & $(-5) 1.40288$ & $(-4) 1.81213$ & $(-3) 1.08425$ & $(-3) 2.34445$ & $(-3) 6.51070$ & $(-2) 1.41493$ & $(-2) 3$ & 237 & $(-1) 2.15850$ \\
\hline 1.9 & $(-5) 2.43406$ & $(-4) 2.74819$ & $(-3) 1.49697$ & $(-3) 3.10904$ & $(-3) 8.18863$ & $(-2) 1.71032$ & $(-2) 3$ & 2995 & 9192 \\
\hline 2 & $(-5) 4.00012$ & $(-4) 4.00120$ & $(-3) 2.00301$ & $(-3) 4.01206$ & $(-2) 1.00760$ & $(-2) 2.03083$ & $(-2) 4.12685$ & $(-1) 1.08699$ & 2142 \\
\hline 2.2 & $(-5) 9.45458$ & $(-4) 7.67259$ & $(-3) 3.32018$ & $(-3) 6.24792$ & $(-2) 1.44563$ & $(-2)^{2} .74015$ & $(-2) 5.24003$ & $(-1) 1.27452$ & $(-1)^{2} .66904$ \\
\hline 2.5 & $(-4) 2.66562$ & $(-3) 1.68348$ & $(-3) 6.11887$ & $(-2) 1.06862$ & $(-2) 2.24181$ & $(-2) 3.94858$ & $(-2) 7.02107$ & $(-1) 1.55155$ & 01369 \\
\hline 3 & $(-4) 9.54251$ & $(-3) 4.43853$ & $(-2) 1.30458$ & $(-2) 2.08065$ & $(-2) 3.87476$ & $(-2) 6.24125$ & $(-1) 1.01537$ & 9369 & 2452 \\
\hline 3.5 & $(-3) 2.39163$ & $(-3) 8$ & $(-2)$ & $(-2) 3.38123$ & 8523 & 74291 & $(-1$ & 0543 & $1-1$ \\
\hline 4 & $(-3) 4.79240$ & $(-2) 1.52347$ & $(-2) 3.43991$ & $(-2) 4.90140$ & $(-2) 7.87055$ & $(-1) 1.13378$ & $(-1) 1.64929$ & 78522 & $(-1) 4.35554$ \\
\hline 4.5 & $(-3) 8.26707$ & $(-2) 2.31640$ & $(-2) 4.79234$ & $(-2) 6.57742$ & $(-1) 1.00527$ & $(-1) 1.39492$ & $(-1) 1.95398$ & 13422 & $(-1) 4.69705$ \\
\hline 5 & $(-2) 1.28351$ & $(-2) 3.25178$ & $(-2) 6.27368$ & $(-2) 8.35630$ & $(-1) 1.22754$ & $(-1) 1.65280$ & $(-1) 2.24569$ & $(-1) 3.45464$ & $(-1) 5.00000$ \\
\hline 6 & $(-2) 2.50279$ & $(-2) 5.45389$ & $(-2) 9.47591$ & $(-1) 1.20653$ & $(-1) 1.66955$ & $(-1) 2.14770$ & $(-1) 2.78583$ & 1981 & 1335 \\
\hline 7 & $(-2) 4.06312$ & $(-2) 7.95192)$ & $(-1) 1.28181$ & $(-1) 1.58006$ & 09417 & 60634 & $(-1) 3.26847$ & 0008 & $(-1$ \\
\hline 8 & $(-2) 5.87480$ & $(-1) 1.06068$ & $(-1) 1.61587$ & $(-1) 1.94368$ & $(-1) 2.49327$ & $(-1) 3.02601$ & $(-1) 3.69818$ & 91174 & $(-1) 6.27868$ \\
\hline 9 & $(-2) 7.85603$ & $(-1) 1.33198$ & $(-1) 1.94151$ & $(-1) 2.29097$ & $(-1) 2.86423$ & $(-1) 3.40802$ & $(-1) 4.08107$ & 6776 & 57136 \\
\hline 10 & $(-2) 9.94020$ & 60247 & $(-1) 2.25419$ & $(-1) 2.61906$ & $(-1) 3.20714$ & $(-1) 3.75528$ & $(-1) 4.42317$ & 7830 & 2144 \\
\hline 12 & $(-1) 1.42256$ & $(-1) 2.12538$ & $(-1) 2.83315$ & $(-1) 3.21529$ & $(-1) 3.81489$ & $(-1) 4.35898$ & $(-1) 5.00623$ & 9304 & 621 \\
\hline 15 & $(-1) 2.05183$ & $(-1) 2.83892$ & $(-1) 3.58331$ & $(-1) 3.97056$ & $(-1) 4.56176$ & $(-1) 5.08363$ & $(-1) 5.68934$ & $(-1) 6.67584$ & $(-1) 7.6$ \\
\hline 20 & $(-1) 2.98775$ & $(-1) 3$ & $(-1) 4.56539$ & $(-1) 4.93664$ & $(-1) 5.4$ & $(-1) 5.96046$ & $(-1) 6.49538$ & $(-1) 7.33951$ & $(-1) \varepsilon$ \\
\hline 24 & $(-1) 3.62052$ & $(-1) 4.45402$ & $(-1) 5.16925$ & $(-1) 5.52037$ & $(-1) 6.03408$ & $(-1) 6.46843$ & $(-1) 6.95346$ & $(-1) 7.70656$ & $(-1)$ \\
\hline 30 & $(-1) 4.40136$ & $(-1) 5.20205$ & $(-1) 5.86649$ & $(-1) 6.18619$ & $(-1) 6.64712$ & $(-1) 7.03107$ & $(-1) 7.45411$ & $(-1) 8.10016$ & $(-1) 8.70803$ \\
\hline 40 & $(-1) 5.36959$ & $(-1) 6.09372$ & $(-1) 6.67443$ & $(-1) 6.94816$ & $(-1) 7.33694$ & $(-1) 7.65589$ & $(-1) 8.00254$ & 2303 & $(-1)$ \\
\hline 60 & $(-1) 6.57659$ & $(-1) 7.16139$ & $(-1) 7.61424$ & $(-1) 7.82325$ & $(-1) 8.11561$ & $(-1) 8.35174$ & $(-1) 8.60480$ & $(-1) 8.97822$ & $(-1) 9.31665$ \\
\hline 120 & 005 & $(-1) 8.44604$ & $(-1) 8.71199$ & $(-1) 8.83212$ & $(-1) 8.99753$ & $(-1) 9.12900$ & $(-1) 9.26789$ & $(-1) 9.46922$ & $(-1) 9.64816$ \\
\hline$\infty$ & 1.00000 & 1.00000 & 1.00000 & 1.00000 & 1.00000 & 1.00000 & 1.00000 & 1.00000 & 1.00000 \\
\hline
\end{tabular}

The numbers in parentheses indicate the power of ten by which the number following is to be multiplied, e.g., $(-1) 1.23456=0.123456$. 


\begin{tabular}{|c|c|c|c|c|c|c|c|c|c|}
\hline$\nu_{2}$ & $p=0.0001$ & $p=0.001$ & $p=0.005$ & $p=0.01$ & $p=0.025$ & $p=0.05$ & $p=0.1$ & $p=0.25$ & $p=0.5$ \\
\hline I & $(-9) 2.84444$ & $(-7) 2.84445$ & $(-6) 7.11118$ & $(-5) 2.84455$ & $(-4) 1.77820$ & $(-4) 7.11786$ & 85530 & .82151 & 0328 \\
\hline 1.1 & $(-8) 1.54660 k$ & $(-6) 1.01756$ & $(-5) 1.89856$ & $(-5) 6.69545$ & $(-4) 3.54379$ & $(-3) 1.25112$ & $(-3) 4.43003$ & $(-2) 2.40402$ & $(-2) 9.26681$ \\
\hline 1.2 & $(-8) 6.35664)$ & $(-6) 2.95050$ & $(-5) 4.31388$ & $(-4) 1.36973$ & $(-4) 6.31157$ & $(-3) 2.00725$ & $(-3) 6.40778$ & $(-2) 3.04090$ & $(-1) 1.06225$ \\
\hline 1.3 & $(-7) 2.10639$ & $(-6) 7.27787$ & $(-5) 8.65734$ & $(-4) 2.51533$ & $(-3) 1.03091$ & $(-3) 3.00179$ & $(-3) 8.78111$ & $(-2) 3.72213$ & $(-1) 1.19624$ \\
\hline 1.4 & $(-7) 5.89265$ & $(-5) 1.58085$ & $(-4) 1.57576$ & $(-4) 4.24297$ & $(-3) 1.57305$ & $(-3) 4.24769$ & $(-2) 1.15325$ & $(-2) 4.43902$ & $(-1) 1.32813$ \\
\hline 1.5 & $(-6) 1.43950$ & $(-5) 3.10142$ & $(-4) 2.65239$ & $(-4) 6.68669$ & $(-3) 2.27297$ & $(-3) 5.75036$ & $(-2) 1.46383$ & $(-2) 5.18412$ & $(-1) 1.45757$ \\
\hline 1.6 & $(-6) 3.14957$ & $(-5) 5.60114$ & $(-4) 4.18952$ & $(-4) 9.97081$ & $(-3) 3.14189$ & $(-3) 7.50911$ & $(-2) 1.80713$ & $(-2) 5.95113$ & $(-1) 1.58435$ \\
\hline 1.7 & $(-6) 6.29278$ & $(-5) 9.44831$ & $(-4) 6.27946$ & $(-3) 1.42052$ & $(-3) 4.18709$ & $(-3) 9.51860$ & $(-2) 2.18031$ & $(-2) 6.73473$ & $(-1) 1.70833$ \\
\hline 1.8 & $(-5) 1.16557$ & $(-4) 1.50561$ & $(-4) 9.00924$ & $(-3) 1.94825$ & $(-3) 5.41235$ & $(-2) 1.17700$ & $(-2) 2.58051$ & $(-2) 7.53047$ & $(-1) 1.82944$ \\
\hline 1.9 & $(-5) 2.02538$ & $(-4) 2.28682$ & $(-3) 1.24578$ & $(-3) 2.58770$ & $(-3) 6.81846$ & $(-2) 1.42522$ & $(-2) 3.00498$ & $(-2) 8.33460$ & 4766 \\
\hline 2 & $(-5) 3.33344$ & $(-4) 3.33445$ & $(-3) 1.66945$ & $(-3) 3.34451$ & $(-3) 8.40376$ & $(-2) 1.69524$ & $(-2) 3.45106$ & $(-2) 9.14397$ & $(1-1) 2.06299$ \\
\hline 2.2 & $(-5) 7.90157$ & $(-4) 6.41264$ & 7554 & 2427 & $(-2)$ & $(-2) 2.29522$ & 9840 & $(-1) 1.07683$ & $(-1) 2.28516$ \\
\hline 2.5 & $(-4) 2.23696$ & $(-3) 1.41292$ & $(-3) 5.13727$ & $(-3) 8.97517$ & $(-2) 1.88464$ & $(-2) 3.32406$ & $(-2) 5.92560$ & $(-1) 1.31909$ & $(1-1) 2.59797$ \\
\hline 3 & $(-4) 8.05925$ & $(-3) 3.74960$ & $(-2) 1.10282$ & $(-2) 1.75990$ & $(-2) 3.28198$ & $(-2) 5.29625$ & $(-2) 8.64344$ & $(-1) 1.71128$ & 06947 \\
\hline 3.5 & $(-3) 2.03179$ & $(-3) 7.60454$ & $(-2) 1.92392$ & $(-2) 2.87912$ & $(-2) 4.93499$ & 47483 & 14510 & 8258 & 8650 \\
\hline 4 & $(-3) 4.09365$ & $(-2) 1.30229$ & $(-2) 2.94448$ & $(-2) 4.19986$ & $(-2) 6.75860$ & $(-2) 9.76115$ & $(-1) 1.42559$ & $(-1) 2.43022$ & $(-1) 3.85728$ \\
\hline 4.5 & $(-3) 7.09785$ & $(-2) 1.99078$ & $(-2) 4.12565$ & $(-2) 5.66941$ & $(-2) 8.68633$ & $(-1) 1.20875$ & $(-1) 1$ & 5402 & 18874 \\
\hline 5 & $(-2) 1.10728$ & $(-2) 2.80889$ & $(-2) 5.43009$ & $(-2) 7.24286$ & $(-1) 1.06687$ & $(-1) 1.44085$ & $(-1) 1.96641$ & $(-1) 3.05496$ & $(-1) 4.48665$ \\
\hline 6 & $(-2) 2.17821$ & $(-2) 4.75519$ & $(-2) 8$ & $(-1) 1$ & 46633 & $(-1) 1.89255$ & $(-1) 2.46636$ & 9436 & $(-1) 5.00000$ \\
\hline 7 & $(-2) 3.56417$ & $(-2) 6.99126$ & $(-1) 1.13027$ & $(-1) 1.39586$ & $(-1) 1.85619$ & 1820 & 101 & 136 & 2634 \\
\hline 8 & $(-2) 5.19012$ & $(-2) 9.39539$ & $(-1) 1.43596$ & $(-1) 1.73070$ & $(-1) 2.22778$ & $(-1) 2.71338$ & $(-1)^{3}$ & 802 & 8593 \\
\hline 9 & $(-2) 6.98524$ & $(-1) 1.18782$ & $(-1) 1.73735$ & $(-1) 2.05430$ & $(-1) 2.57744$ & $(-1) 3.07771$ & $(-1) 3.70292$ & $(-1) 4.82448$ & $(-1) 6.09323$ \\
\hline 10 & $(-2) 8.89021$ & $(-1) 1.43774$ & $(-1) 2.02972$ & $(-1) 2.36324$ & $\mid(-1) 2.90421$ & $(-1) 3.41261$ & $(-1) 4.03820$ & $(-1) 5.13903$ & $(-1) 6.35884$ \\
\hline 12 & $(-1) 1.28533 \mid$ & $(-1) 1.92700$ & $(-1) 2.57831$ & $(-1) 2.93230$ & $(-1) 3.49144$ & $(-1) 4.00311$ & $(-1) 4$ & 6795 & 9481 \\
\hline 15 & $(-1) 1.87696$ & $(-1) 2.60642$ & $(-1) 3.30216$ & $(-1) 3.66662$ & $(-1) 4.22678$ & $(-1) 4.72548$ & $(-1) 5.31002$ & .27824 & 3296 \\
\hline 20 & $(-1) 2.77495$ & $(-1) 3$ & $(-1) 4.27050$ & $(-1) 4.62657$ & $(-1) 5$ & $(-1) 5.61895$ & $(-1) 6.14478$ & $(-1) 6$ & 314 \\
\hline 24 & $(-1) 3.39283$ & $(-1) 4.18831$ & $(-1) 4.87693$ & $(-1) 5.21736$ & $(-1) 5.71871$ & $(-1) 6.14610$ & 62786 & $(-1) 7.38781$ & 13526 \\
\hline 30 & $(-1) 4.16608$ & $(-1) 4.93961$ & $(-1) 5.58708$ & $(-1) 5.90077$ & $(-1) 6.35591$ & $(-1) 6.73807$ & $(-1) 7.16298$ & $(-1) 7.82189$ & $(-1) 8.45782$ \\
\hline 40 & $(-1) 5$ & $(-1)$ & $(-1) 6$ & 69500 & $(-1) 7.08387$ & $(-1) 7.40533$ & $(-1) 7.75776$ & 9472 & $(-1) 8.80296$ \\
\hline 60 & $(-1) 6.37631 \mid$ & $(-1) 6.95795$ & $(-1) 7.41189$ & $(-1) 7.62272$ & $(-1) 7.91931$ & $(-1) 8.16057$ & $(-1) 8.42125$ & $(-1) 8.81129$ & 7310 \\
\hline 120 & $(-1) 7.95788$ & $(-1) 8.31817$ & $(-1) 8.58924$ & $(-1) 8.71237$ & $(-1) 8$ & $(-1) 9.01916$ & $(-1) 9.16430$ & $(-1) 9.37742$ & $(-1) 9.57104$ \\
\hline$\infty$ & 1.00000 & 0000 & 1.00000 & 1.00000 & 1.00000 & 1.00000 & 1.00000 & 2000 & 1.00000 \\
\hline
\end{tabular}




\begin{tabular}{|c|c|c|c|c|c|c|c|c|c|}
\hline$\nu_{2}$ & $p=0.0001$ & $p=0.001$ & $p=0.005$ & $p=0.01$ & $p=0.025$ & $p=0.05$ & $p=0.1$ & $p=0.25$ & $p=0.5$ \\
\hline 1 & $(-9) 2.40957$ & $(-7) 2.40957$ & $(-6) 6.02399$ & $(-5) 2.40967$ & $(-4) 1.50636$ & $(-4) 6.02999$ & $(-3) 2.41931$ & $(-2) 1.54525$ & $(-2) 6.73783$ \\
\hline 1.1 & $(-8) 1.31183$ & $(-7) 8.63098$ & $(-5) 1.61037$ & $(-5) 5.67912$ & $(-4) 3.00593$ & $(-3) 1.06130$ & $(-3) 3.75886$ & $(-2) 2.04295$ & $(-2) 7.91884$ \\
\hline 1.2 & $(-8) 5.39850$ & $(-6) 2.50577$ & $(-5) 3.66366$ & $(-4) 1.16328$ & $(-4) 5.36048$ & $(-3) 1.70496$ & $(-3) 5.44462$ & $(-2) 2.58866$ & $(-2) 9.09825$ \\
\hline 1.3 & $(-7) 1.79110$ & $(-6) 6.18849$ & $(-5) 7.36152$ & $(-4) 2.13886$ & $(-4) 8.76667$ & $(-3) 2.55306$ & $(-3) 7.47173$ & $(-2) 3.17410$ & $(-1) 1.02691$ \\
\hline 1.4 & $(-7) 5.01668$ & $(-5) 1.34585$ & $(-4) 1.34153$ & $(-4) 3.61235$ & $(-3) 1.33937$ & $(-3) 3.61741$ & $(-3) 9.82672$ & $(-2) 3.79202$ & $(-1) 1.14265$ \\
\hline 1.5 & $(-6) 1.22697$ & $(-5) 2.64352$ & $(-4) 2.26082$ & $(-4) 5.69971$ & $(-3) 1.93771$ & $(-3) 4.90345$ & $(-2) 1.24907$ & $(-2) 4.43618$ & $(-1) 1.25672$ \\
\hline 1.6 & $|(-6) 2.68767|$ & $(-5) 4.77973$ & $(-4) 3.57522$ & $(-4) 8.50918$ & $(-3) 2.68174$ & $(-3) 6.41145$ & $(-2) 1.54418$ & $(-2) 5.10128$ & $(-1) 1.36891$ \\
\hline 1.7 & $(-6) 5.37605$ & $(-5) 8.07194$ & $(-4) 5.36492$ & $(-3) 1.21371$ & $(-3) 3.57821$ & $(-3) 8.13763$ & $(-2) 1.86569$ & $(-2) 5.78282$ & $(-1) 1.47907$ \\
\hline 1.8 & $(-6) 9.96883$ & $(-4) 1.28773$ & $(-4)$ & $(-3) 1.6$ & $(-3) 4.63088$ & $(-2) 1.00753$ & $(-2) 2.21124$ & $(-2) 6.47697$ & $(-1) 1.58710$ \\
\hline 1.9 & $(-5) 1.73417$ & $(-4) 1.95805$ & $(-3) 1.06676$ & $(-3) 2.21605$ & $(-3) 5.84096$ & $(-2) 1.22156$ & $(-2) 2.57855$ & $(-2) 7.18053$ & $(-1) 1.69297$ \\
\hline 2 & $(-5) 2.85724$ & $(-4) 2.85816$ & $(-3) 1.43113$ & $(-3) 2.86741$ & $(-3) 7.20756$ & $(-2) 1.45484$ & $(-2) 2.96544$ & $(-2) 7.89076$ & $(-1) 1$ \\
\hline 2.2 & $(-5) 6.78707$ & $(-4) 5.50837$ & $(-3) 2.38451$ & $(-3) 4.48902$ & $(-2) 1.03986$ & $(-2) 1.97467$ & $(-2) 3.78985$ & $(-2) 9.32237$ & 99744 \\
\hline 2.5 & $(-4) 1.92726$ & $(-3) 1.21741$ & $(-3) 4.42754$ & $(-3) 7.73725$ & $(-2) 1.62580$ & $(-2) 2.87042$ & $(-2) 5.12633$ & $(-1) 1.14726$ & $(-1) 2.28264$ \\
\hline 3 & $(-4) 6.97649$ & $(-3) 3.24647$ & $(-3) 9.55296$ & $(-2) 1.52515$ & $(-2) 2.84709$ & $(-2) 4.60072$ & $(-2) 7.52571$ & $(-1) 1.49913$ & $(-1) 2.71807$ \\
\hline 3.5 & $(-3) 1.76661 \mid$ & $(-3) 6.61434$ & $(-2) 1.67464$ & $(-2) 2.50761$ & $(-2) 4.30394$ & $(-2) 6.52994$ & $(-1) 1.00304$ & $(-1) 1.83647$ & $(-1)$ \\
\hline 4 & $(-3) 3.57412$ & $(-2) 1.13764$ & $(-2) 2.57480$ & $(-2) 3.67545$ & $(-2) 5.92426$ & $(-2) 8.57275$ & $(-1) 1.25578$ & $(-1) 2,15599$ & $(-1) 3.46086$ \\
\hline 4.5 & $(-3) 6.22131$ & $(-2) 1.74626$ & $(-2) 3.62353$ & $(-2) 4.98408$ & $(-2) 7.65054$ & $(-1) 1.06691$ & $(-1) 1.50571$ & $(-1) 2.45676$ & $(-1) 3,77925$ \\
\hline 5 & $(-3) 9.74136$ & 47355 & $(-2) 4.78913$ & $(-2) 6.39480$ & $(-2) 9.43903$ & $(-1) 1.27776$ & $(-1) 1,74976$ & $(-1) 2.73904$ & $(-1) 4.06845$ \\
\hline 6 & $(-2) 1.92948$ & 811 & $(-2) 7.36184 \mid$ & $(-2) 9$ & $(-1) 1.30809$ & $(-1) 1.69269$ & $(-1) 2.21392$ & $(-1) 3.25157$ & 7366 \\
\hline 7 & $(-2) 3.17693$ & $(-2) 6.24276$ & $(-1) 1.01159$ & $(-1) 1.25112$ & $(-1) 1.66808$ & $(-1) 2.08897$ & $(-1) 2.64206$ & $(-1) 3.70213$ & $(-1) 5$ \\
\hline 8 & $(-2) 4.65261$ & $(-2) 8.44004$ & $(-1) 1.29326$ & $(-1) 1.56118$ & $(-1) 2.01512$ & $(-1) 2.46129$ & $(-1) 3.03387$ & $(-1) 4.09965$ & $(-1) 5.36446$ \\
\hline 9 & $(-2) 6.29451$ & 07289 & $(-1) 1.5$ & $(-1) 1.86374$ & $(-1) 2.34501$ & $(-1) 2.80818$ & $(-1) 3.39147$ & $(-1) 4.45207$ & $(-1) 5.67954$ \\
\hline 10 & $(-2) 8.04945$ & 30511 & $(-1) 1.84783$ & $(-1) 2.15513$ & $(-1) 2.65613$ & $(-1) 3.13006$ & $(-1) 3.71778$ & $(-1) 4.76616)$ & $(-1) 5.95459$ \\
\hline 12 & $(-1) 1.17363$ & $(-1) 1$ & $(-1) 2$ & $(-1) 2.6$ & $(-1) 3.22186$ & $(-1) 3$ & $(-1) 4.28886$ & $(-1) 5.30087$ & $(-1)$ \\
\hline 15 & $(-1) 1.73177$ & $(-1) 2.41213$ & $(-1) 3.06555$ & $(-1) 3.40980$ & $(-1) 3.94182$ & $(-1) 4.41873$ & $(-1) 4.98210$ & $(-1) 5.92817$ & $(-1) 6$ \\
\hline 20 & $(-1) 2.59391 \mid$ & 4203 & 01621 & 35814 & 87188 & $(-1) 5.31942$ & $(-1) 5.83466$ & $(-1) 6.67199$ & $(-1)$ \\
\hline 24 & $(-1) 3.19633$ & $(-1) 3.95750$ & 128 & $(-1) 4.95136$ & $(-1) 5.44012$ & $(-1) 5.85960$ & $(-1) 6.33607$ & $(-1) 7.09734$ & 6185 \\
\hline 30 & $(-1) 3$ & $(-1)$ & $(-1)$ & $(-1) 5$ & 9481 & $(-1) 6.47383$ & $(-1) 6.89839$ & $(-1) 7.56494$ & $(-1) 8.22136$ \\
\hline 40 & $(-1) 4.93534$ & $(-1) 5.62993$ & $(-1) 6$ & $(-1) 6.46556$ & $(-1) 6$ & $(-1) 7.17577$ & $(-1) 7.53187$ & $(-1) 8.08089$ & $(-1) 8.61071$ \\
\hline 60 & $(-1) 6.19382$ & $(-1) 6.77163$ & $(-1) 7.22563$ & $(-1) 7.43759$ & $(-1) 7.73719$ & $(-1) 7.98235$ & $(-1) 8.24904$ & $(-1) 8.65253$ & $(-1) 9.03376$ \\
\hline 120 & .83464 & $(-1) 8.19847$ & $(-1) 8,47385$ & $(-1) 8.59954$ & $(-1) 8.77427$ & $(-1) 8.91482$ & $(-1) 9.06534$ & $(-1) 9.28866$ & $(-1) 9.49506$ \\
\hline$\infty$ & 1.00000 & 1.00000 & 1.00000 & 1.00000 & 1.00000 & 1.00000 & 1.00000 & 1.00000 & 1.00000 \\
\hline
\end{tabular}




\begin{tabular}{|c|c|c|c|c|c|c|c|c|c|}
\hline$\nu_{2}$ & $p=0.0001$ & $p=0.001$ & $p=0.005$ & $p=0.01$ & $p=0.025$ & $p=0.05$ & $p=0.1$ & $p=0.25$ & $p=0.5$ \\
\hline I & $(-9) 2.08980$ & $(-7) 2.08980$ & $(-6) 5.22454$ & $(-5) 2.08988$ & $(-4) 1.30646$ & $(-4) 5.22996$ & $(-3) 2.09858$ & $(-2) 1.34159$ & $(-2) 5.87108$ \\
\hline I.I & $(-8) 1.13882$ & $(-7) 7.49271$ & $(-5) 1.39799$ & $(-5) 4.93017$ & $(-4) 2.60956$ & $(-4) 9.21402$ & $(-3) 3.26396$ & $(-2) 1.77597$ & $(-2) 6.91226$ \\
\hline 1.2 & $(-8) 4.69093$ & $(-6) 2.17735$ & $(-5) 3.18348$ & $(-4) 1.01082$ & $(-4) 4.65806$ & $(-3) 1.48166$ & $(-3) 4.73273$ & $(-2) 2.25329$ & $(-2) 7.95548$ \\
\hline 1.3 & $(-7) 1.55778$ & $(-6) 5.38234$ & $(-5) 6.40260$ & $(-4) 1.86027$ & $(-4) 7.62511$ & $(-3) 2.22086$ & $(-3) 6.50164$. & $(-2) 2.76649$ & $(-2) 8.99451$ \\
\hline 1.4 & $(-7) 4.36713$ & $(-5) 1.17159$ & $(-4) 1.16784$ & $(-4) 3.14470$ & $(-3) 1.16605$ & $(-3) 3.14978$ & $(-3) 8.55991$ & $(-2) 3.30937$ & $(-1) 1.00250$ \\
\hline 1.5 & $(-6) 1.06905$ & $(-5) 2.30329$ & $(-4) 1.96988$ & $(-4) 4.96633$ & $(-3) 1.68853$ & $(-3) 4.27373$ & $(-2) 1.08920$ & $(-2) 3.87659$ & $(-1) 1.10439$ \\
\hline 1.6 & $(-6) 2.34381$ & $(-5) 4.16823$ & $(-4) 3.11788$ & $(-4) 7.42093$ & $(-3) 2.33905$ & $(-3) 5.59349$ & $(-2) 1.34797$ & $(-2) 4.46357$ & $(-1) 1.20491$ \\
\hline 1.7 & $(-6) 4.69229$ & $(-5) 7.04533$ & $(-4) 4.68273$ & $(-3) 1.05942$ & $(-3) 3.12383$ & $3(-3) 7.10634$ & $(-2) 1.63035$ & $(-2) 5.06642$ & $(-1) 1.30391$ \\
\hline 1.8 & $(-6) 8.70831$ & $(-4) 1.12491$ & $(-4) 6.73184$ & $(-3) 1.45596$ & $(-3) 4.04651$ & $(1-3) 8.80695$ & $(-2) 1.93437$ & $(-2) 5.68184$ & $(-1) 1.40130$ \\
\hline 1.9 & $(-5) 1.51616$ & $(-4) 1.71191$ & $(-4) 9.32711$ & $(-3) 1.93773$ & $(-3) 5.10853$ & $(-2) 1.06881$ & $(-2) 2.25808$ & $(-2) 6.30700$ & $(-1) 1.49702$ \\
\hline 2 & $(-5) 2.50009$ & $(-4) 2.50094$ & $(-3) 1.25235$ & $(-3) 2.50943$ & $(-3) 6.30946$ & $(-2) 1.27415$ & $(-2) 2.59963$ & $(-2) 6.93951$ & $(-1) 1.59104$ \\
\hline 2.2 & $(-5) 5.94824$ & $(-4) 4.82772$ & $(-3) 2.09010$ & $(-3) 3.93528$ & $(-3) 9.11927$ & $7(-2) 1.73273$ & $(-2) 3.32930$ & $(-2) 8.21875$ & $(-1) 1.77389$ \\
\hline 2.5 & $(-4) 1.69299$ & $(-3) 1.06949$ & $(-3) 3.89034$ & $(-3) 6.79984$ & $(-2) 1.42956$ & $(-2) 2.52587$ & $(-2) 4.51730$ & $(-1) 1.01506$ & $(-1) 2.03537$ \\
\hline 3 & $(-4) 6.15093$ & $(-3) 2.86273$ & $(-3) 8.42686$ & $(-2) 1.34581$ & $(-2) 2.51427$ & $(-2) 4.06714$ & $(x-2) 6.66469$ & $(-1) 1.33387$ & $(-1)^{2} .43864$ \\
\hline 3.5 & $(-3) 1.56292$ & $(-3) 5.85331$ & $(-2) 1.48281$ & $(-2) 2.22142$ & $(-2) 3.81666$ & $(-2) 5.79813$ & $(-2) 8.92487$ & 64255 & $(-1) 2.80480$ \\
\hline 4 & $(-3) 3.17233$ & $(-2) 1.01018$ & $(-2) 2.28812$ & $(-2) 3.26821$ & $(-2) 5.27450$ & $(-2) 7.64404$ & $(-1) 1.12235$ & $(-1) 1.93764$ & $(-1) 3.13810$ \\
\hline 4.5 & $(-3) 5.53902$ & $(-2) 1.55567$ & $(-2) 3.23129$ & $(-2) 4.44782$ & $(-2) 6.83739$ & $(-2) 9.55126$ & $(-1) 1.35136$ & $(-1) 2.21779$ & $(-1) 3.44246$ \\
\hline 5 & $(-3) 8.69860$ & $(-2) 2.21047$ & $(-2) 4.28494$ & $(-2) 5.72643$ & $(-2) 8.46634$ & $(-1) 1.14820$ & $(-1) 1.57658$ & $(-1) 2.48281$ & $(-1) 3.72132$ \\
\hline 6 & $(-2) 1.73247$ & $(1-2) 3.79164$ & $(-2) 6.62792$ & $(-2) 8.47300$ & 18117 & $(-1) 1.53161$ & $(-1) 2.00909$ & 96917 & $(-1) 4.21407$ \\
\hline 7 & $(-2) 2.86702$ & $(-2) 5.64188$ & $(-2) 9.15932$ & $(-1) 1.13415$ & $(-1) 1.51532$ & $(-1) 1.90187$ & $(-1) 2.41274$ & 40219 & 63554 \\
\hline 8 & $(-2) 4.21841$ & $(-2) 7.66545$ & $(-1) 1.17704$ & $(-1) 1.42270$ & $(-1) 1.84052$ & $(-1) 2.25322$ & $(-1) 2.78602$ & $(-1) 3.78848$ & $(-1) 5.00000$ \\
\hline 9 & $(-2) 5.73173$ & $(-2) 9.78857$ & $(-1) 1.43893$ & $(-1) 1.70658$ & $(-1) 2.15230$ & $(-1) 2.58349$ & $(-1) 3.12986$ & $(-1) 4.13430$ & $(-1) 5.31822$ \\
\hline 10 & $(-2) 7.35904$ & $(-1) 1.19570$ & $(-1) 1.69700$ & $(-1) 1.98202$ & $(-1) 2.44863$ & $(-1) 2.89241$ & 44623 & 44514 & $(-1) 5.59845$ \\
\hline 12 & $(-1) 1.08064$ & $(-1) 1.62860$ & $(-1) 2.19136$ & $(-1) 2.50026$ & $(-1) 2.99295$ & $(-1) 3.44941$ & 00580 & 98008 & 06915 \\
\hline 15 & $(-1) 1.60881$ & .24667 & $(-1) 2.86288$ & 8907 & 550 & 209 & 9509 & 61693 & 61269 \\
\hline 20 & $(-1) 2.43730$ & $(-1) 3.14862$ & $(-1) 3.79364$ & $(-1) 4.12241$ & $(-1) 4.61868$ & $(-1) 5.05350$ & $(-1) 5.55737$ & $(-1) 6.38515$ & $(-1) 7.24724$ \\
\hline 24 & $(-1) 3.02417$ & $(-1) 3.75412$ & $(-1) 4.39472$ & $(-1) 4.71486$ & $(-1) 5.19109$ & $(-1) 5.60216$ & $(-1) 6.07207$ & $(-1) 6.83089$ & .60607 \\
\hline 30 & $(-1) 3.77637)$ & $(-1) 4.50103$ & $(-1) 5.11592$ & $(-1) 5.41702$ & $(-1) 5.85823$ & $(-1) 6.23321$ & $(-1) 6.65587$ & $(-1) 7.32633$ & $(-1) 7.99762$ \\
\hline 40 & $(-1) 4.75026$ & $(-1) 5.43063$ & $(1-1) 5.98817$ & $(-1) 6.25549$ & $(-1) 6.64111$ & $(-1) 6.96362$ & $(-1) 7.32186$ & $(-1) 7.87966$ & $(-1) 8.42657$ \\
\hline 60 & $(-1) 6.02580$ & $(-1) 6.59931$ & $(-1) 7.05256$ & $(-1) 7.26513$ & $(-1) 7.56684$ & 1) 7.81497 & $(-1) 8$ & $(-1) \varepsilon$ & 9851 \\
\hline 120 & $(-1) 7.71869$ & $(-1) 8.08543$ & $(-1) 8.36449$ & $(-1) 8.49237$ & $(-1) 8.67082$ & $(-1) 8.81501$ & $(-1) 8$. & $(-1) 9.20252$ & $(-1) 9.42023$ \\
\hline$\infty$ & 1.00000 & 1.00000 & 1.00000 & 1.00000 & 1.00000 & 1.00000 & 1.00000 & 1.00000 & 1.00000 \\
\hline
\end{tabular}


PERCENTAGE POINTS OF THE BETA DISTRIBUTION

$\nu_{1}=$

\begin{tabular}{|c|c|c|c|c|c|c|c|c|c|}
\hline$\nu_{2}$ & $p=0.0001$ & $p=0.001$ & $p=0.005$ & $p=0.01$ & $p=0.025$ & $p=0.05$ & $p=0.1$ & $p=0.25$ & $p=0.5$ \\
\hline 1 & $(-9)$ & $(-7) 1.84483$ & $(-6) 4.61212$ & $(-5) 1.84491$ & $(-4) 1.15333$ & $(-4) 4.61704$ & $(-3) 1.85282$ & $(-2) 1.18529$ & $9(-2)$ \\
\hline 1.1 & $(-8) 1$ & $(-7) 6.61929$ & $(-5) 1.23503$ & $(-5) 4.35547$ & $(-4) 2.30540$ & $(-4) 8.14039$ & $(-3) 2.88404$ & $(-2) 1.57062$ & $(-2) 6$ \\
\hline 1.2 & $(-8) 4.14712$ & $(-6) 1.92493$ & $(-5) 2.81443$ & $(-5) 8.93645$ & $(-4) 4.11817$ & $(-3) 1.31001$ & $(-3) 4.18525$ & $(-2) 1.99474$ & $4(-2) 7$ \\
\hline 1.3 & $(-7) 1.37817$ & $(-6) 4.76178$ & $(-5) 5.66443)$ & $(-4) 1.64581$ & $(-4) 6.74627$ & $(-3) 1.96506$ & $(-3) 5.75422$ & $(-2) 2.45153$ & $3(-2) 8.00079$ \\
\hline 1.4 & $(-7) 3.86634$ & $(-5) 1.03724$ & $(-4) 1.03393$ & $(-4) 2.78414$ & $(-3) 1.03241$ & $(-3) 2.78910$ & $(-3) 7.58211$ & $(-2) 2.93557$ & $7(-2)$ \\
\hline 1.5 & $(-7) 9.47121$ & $(-5) 2.04059$ & $(-4) 1.74522$ & $(-4) 4.40001$ & $(-3) 1.49609$ & $(k-3) 3.78722$ & $(-3) 9.65579$ & $(-2) 3.44221$ & $(-2)$ \\
\hline 1.6 & $(-6) 2.07790$ & $(-5) 3.69535$ & $(-4) 2.76420$ & 7930 & $(-3) 2.07396$ & $(-3) 4$ & $(-2) 1$ & $(-2)$ & $4(-1)$ \\
\hline 1.7 & $(-6) 4.16274$ & $(-5) 6.25026$ & $(-4) 4.15437$ & $(-4) 9.39917$ & $(-3) 2.77178$ & $(-3) 6.30691$ & $(-2) 1.44771$ & $(-2) 4$ & $3(-1) 1$ \\
\hline 1.8 & $(-6) 7.73067$ & $(-5) 9.98624$ & $(-4) 5.97632$ & $(-3) 1.29261$ & $(-3) 3.59305$ & $(-3) 7.82213$ & $(-2) 1.71909$ & $(-2)$ & $6(-1)$ \\
\hline 1.9 & $(-5) 1.34683$ & $(-4) 1.52073$ & $(-4) 8.28585$ & $(-3) 1.72150$ & $(-3) 4.53929$ & $(-3) 9.50014$ & 00844 & 2285 & $5(-1)$ \\
\hline 2 & $(-5) 2.22231$ & $(-4) 2.22309$ & $(-3) 1.11328$ & $(-3) 2.23092$ & $(-3) 5.61038$ & $(-2) 1.13338$ & $(-2) 2.31415$ & $(-2)$ & $7 \mid-1$ \\
\hline 2.2 & $(-5) 5.29402$ & $(-4) 4.29684$ & $(-3) 1.86043$ & $(-3) 3.50320$ & $(-3)$ & $(-2) 1$ & $(-2)^{2}$ & $(-2)$ & $7(-1)$ \\
\hline 2.5 & $(-4) 1$ & $(-4) 9.5$ & $(-3$ & 524 & $(-2)$ & $(-2)$ & $(-2)$ & 200 & $0<-1$ \\
\hline 3 & $(-4) 5.50048$ & $(-3) 2$ & $(-3)$ & $(-2) 1$ & $(-2)^{2}$ & $(-2)^{3}$ & $(-2)$ & $(-1$ & $8)(-1$ \\
\hline 3.5 & $(-3) 1.40150$ & $(-3) 5.24991$ & $(-2) 1.33055$ & 99407 & $(-2)$ & $(-2) 5$ & 3964 & $(-$ & \\
\hline 4 & $(-3) 2.85216$ & $(-3) 9.08532$ & $(-2) 2.05918$ & $(-2) 2.94264$ & $(-2) 4.75386$ & $(-2) 6.89786$ & $(-1) 1.01469$ & $(-1) 1$ & $\mid 1-1$ \\
\hline 4.5 & $(-3) 4.99247 \mid$ & $(-2) 1.40283$ & $(-2) 2.91619$ & $(-2) 4.01648$ & $(-2) 6.18158$ & $(-2) 8.64694$ & $(-1) 1.22592$ & 02142 & $2(-1$ \\
\hline 5 & $(-3) 7.85915$ & $(-2) 1.99840$ & $(-2) 3.87763$ & $(-2) 5.18565$ & $(-2) 7.67701$ & $(-1) 1.04271$ & $(-1) 1.43486$ & $(-1) 2$ & $1 k-1$ \\
\hline 6 & $(-2) 1.57239$ & $(-2) 3.44444$ & $(-2) 6.02873$ & 71356 & $(-1) 1.07699$ & $(-1) 1$ & $(-1) 1$ & $(-1)$ & $2(-1$ \\
\hline 7 & 307 & $(-2)$ & 076 & 752 & $(-1$ & $(-1$ & 66 & 77 & \\
\hline 8 & $(-2) 3.85979$ & $(-2) 7.02378$ & $(-1) 1.08039$ & $(-1) 1.30728$ & $(-1) 1.69437$ & $(-1) 2$ & $(-1) 2$ & $(-1) 3$ & $1 \mid(-1$ \\
\hline 9 & $(-2) 5.26357$ & $(-2) 9.00363$ & $(-1) 1.32607$ & $(-1) 1.57452$ & $(-1) 1.98966$ & $(-1) 2.39298$ & $(-1) 2.90669$ & 35966 & $6 \mid(-1$ \\
\hline 10 & $(-2) 6.78088$ & $(-1) 1$ & $(-1) 1.56966$ & $(-1) 1.83548$ & $(-1) 2.27218$ & $(-1)^{2}$ & $(-1)^{3}$ & $(-1)^{4}$ & $4 \mid(-1$ \\
\hline 12 & $(-1)$ & $(-1)$ & $(-1) 2$ & $(-1) 2$ & $(-1)$ & $(-1)$ & $(-1)^{3}$ & $(-1$ & \\
\hline 15 & $(-1) 1.50306$ & $(-1) 2.10370$ & $(-1) 2$ & 682 & & $(-1$ & 104 & & \\
\hline 20 & $(-1) 2.30005$ & $(-1) 2.97827$ & $(-1) 3.59661$ & $(-1) 3$ & $(-1) 4$ & $(-1) 4$ & $(-1) 5$ & $(-1) 6$ & 79 \\
\hline 24 & $(-1) 2.87158$ & $(-1) 3$ & $(-1) 4.19185$ & $(-1) 4.50249$ & $(-1) 4.96644$ & $(-1) 5.36886$ & $(-1) 5.83143$ & $(-1) 6.58518$ & $(-1) 7.36631$ \\
\hline 30 & $(-1) 3$ & $(-1) 4$ & $(-1) 4.91366$ & $(-1) 5.20846$ & 4212 & $(-1) 6$ & $(-1) 6.43216$ & 0377 & $7(-1)$ \\
\hline 40 & $(-1) 4.58152$ & $(-1) 5.24808$ & $(-1) 5.79728$ & $(-1) 6.06170$ & $(-1)$ & $(-1)$ & $(-1) 7.12554$ & $(-1$ & $3(-1$ \\
\hline 60 & $(-1) 5.86991$ & $(-1) 6.43876$ & $(-1) 6.89066$ & $(-1) 7.10344$ & 40652 & 88 & $1-$ & $(-1$ & 0 \\
\hline 120 & $(-1) 7.60888$ & $(-1) 7$ & $(-1) 8.26022$ & $(-1) 8.39001$ & $(-1) 8.57170$ & $(-1) 8.71910$ & $(-1) 8.87848$ & $(-1) 9.11872$ & $(-1) 9.346$ \\
\hline$\propto$ & 1.00000 & 1.00000 & 1.00000 & 1.00000 & 1.00000 & 1.00000 & 1.00000 & 1.00000 & 1.00000 \\
\hline
\end{tabular}

The numbers in parentheses indicate the power of ten by which the number following is to be multiplied, e.g., $(-1) 1.23456=0.123456$. 
PERCENTAGE POINTS OF THE BETA DISTRIBUTION

$\nu_{1}=10$

\begin{tabular}{|c|c|c|c|c|c|c|c|c|c|}
\hline$\nu_{2}$ & $p=0.0001$ & $p=0.001$ & $p=0.005$ & $p=0.01$ & $p=0.025$ & $p=0.05$ & $p=0.1$ & $p=0.25$ & $p=0.5$ \\
\hline 1 & $(-9) 1.65120$ & $(-7) 1.65120$ & $(-6) 4.12804$ & $(-5) 1.65127$ & $(-4) 1.03228$ & $(-4) 4.13254$ & $(-3) 1.65851$ & $(-2) 1.06155$ & 36872 \\
\hline 1.1 & $(-9) 9.01004$ & $(-7) 5.92801$ & $(-5) 1.10605$ & $(-5) 3.90062$ & $(-4) 2.06466$ & $(-4) 7.29057$ & $(-3) 2.58325$ & $(-2) 1.40777$ & $(-2) 5.51009$ \\
\hline 1.2 & $(-8) 3.71617$ & $(-6) 1.72490$ & $(-5) 2.52197$ & $(-5) 8.00786$ & $(-4) 3.69031$ & $(-3) 1.17396$ & $(-3) 3.75116$ & $(-2) 1.78935$ & $(-2) 6.35702$ \\
\hline 1.3 & $(-7) 1.23567$ & $(-6) 4.26940$ & $(-5) 5.07872$ & $(-4) 1.47564$ & $(-4) 6.04890$ & $(-3) 1.76205$ & $(-3) 5.16077$ & $(-2) 2.20088$ & $(-2) 7.20444$ \\
\hline 1.4 & $(-7) 3.46850$ & $(-6) 9.30513$ & $(-5) 9.27548$ & $(-4) 2.49770$ & $(-4) 9.26225$ & $(-3) 2.50247$ & $(-3) 6.8$ & $(-2) 2.63757$ & $(-2) 8.04873$ \\
\hline 1.5 & $(-7) 8.50136$ & $(-5) 1.83164$ & $(-4) 1.56652$ & $(-4) 3.94954$ & $(-3) 1.34299$ & $(-3) 3.40007$ & $(-3) 8.67137$ & $(-2) 3.09530$ & 88730 \\
\hline 1.6 & $(-6) 1.86615$ & $(-5) 3.31877$ & $(-4) 2.48254$ & $(-4) 5.90902$ & $(-3) 1.86281$ & $(-3) 4.45612$ & $(-2) 1.07475$ & $(-2) 3.57049$ & $(-2) 9.71829$ \\
\hline 1.7 & $(-6) 3.74055$ & $(-5) 5.61636$ & $(-4) 3.73311$ & $(-4) 8.44629$ & $(-3) 2.49102$ & $(-3) 5.66908$ & $(-2) 1.30184$ & $(-2) 4.06010$ & $(-1) 1.05404$ \\
\hline 1.8 & $(-6) 6.95033$ & $(-5) 8.97826$ & $(-4) 5.37322$ & $(-3) 1.16221$ & $(-3) 3.23095$ & $(-3) 7.03534$ & $(-2) 1.54692$ & $(-2) 4.56153$ & $(-1) 1,13526$ \\
\hline 1.9 & $(-5) 1.21152$ & $(-4) 1.36795$ & $(-4) 7.45370$ & $(-3) 1.54868$ & $(-3) 4.08417$ & $(-3) 8.54978$ & $(-2) 1.80850$ & $(-2) 5.07255$ & $(-1) 1.21543$ \\
\hline 2 & $(-5) 2.00008$ & $(-4) 2.00080$ & $(-3) 1.00201$ & $(-3) 2.00805$ & $(-3) 5.05076$ & $(-2) 1.02062$ & $(-2) 2.08516$ & $(-2) 5.59125$ & $(-1) 1.29449$ \\
\hline 2.2 & $(-5) 4.76949$ & $(-4) 3.87119$ & $(-3) 1.67625$ & $(-3) 3.15665$ & $(-3) 7.31870$ & $(-2) 1.39174$ & $(-2) 2.67842$ & $(-2) 6.64534$ & $(-1) 1$ \\
\hline 2.5 & $(-4) 1.36202$ & $(-4) 8.60483$ & $(-3) 3.13092$ & $(-3) 5.47401$ & $(-2) 1.15167$ & $(-2) 2.03706$ & $(-2) 3.65032$ & $(-2) 8.24983$ & $(-1) 1$ \\
\hline 3 & $(-4) 4.97467$ & $(-3) 2.31577$ & $(-3) 6.82038$ & $(-2) 1.08977$ & $(-2) 2.03819$ & $(-2) 3.30196$ & $(-2) 5.42455$ & $(-1) 1.09303$ & $(-1)$ \\
\hline 3.5 & $(-3) 1.27039$ & $(-3) 4.75962$ & $(-2) 1.20674$ & 30907 & 11282 & 73775 & 465 & $(-1$ & 564 \\
\hline 4 & $(-3) 2.59093$ & $(-3) 8.25549$ & $(-2) 1.87207$ & $(-2) 2.67632$ & $(-2) 4.32719$ & $(-2) 6.28499$ & $(-2) 9.25953$ & $(-1) 1.61163$ & 64450 \\
\hline 4.5 & $(-3) 4.54463$ & $(-2) 1.27750$ & $(-2) 2.65741$ & $(-2) 3.66183$ & $(-2) 5.64124$ & $(-2) 7.89997$ & $(-1) 1.12191$ & $(-1) 1.85712$ & 2141 \\
\hline 5 & $(-3) 7.16847$ & $(-2) 1.82371$ & $(-2) 3.54153$ & $(-2) 4.73888$ & $(-2) 7.02331$ & $(-2) 9.55098$ & $(-1) 1.31669$ & $(-1) 2.09218$ & 17856 \\
\hline 6 & $(-2) 1.43965$ & $(-2) 3.15608$ & $(-2) 5.52993$ & $(-2) 7.08038$ & $(-2) 9.89883$ & $(-1) 1.28756$ & $(-1) 1.69638$ & $(-1) 2.53074$ & $(-1)$ \\
\hline 7 & $(-2) 2.40098$ & $(-2) 4.73509$ & 70897 & 6276 & 28179 & 1420 & $x-1$ & $(-$ & $(-1)$ \\
\hline 8 & $(-2) 3.55831$ & $(-2) 6.48295$ & $(-2) 9.98672$ & $(-1) 1.20950$ & $(-1) 1.57013$ & $(-1) 1.92903$ & $(-1) 2.39662$ & 9083 & 155 \\
\hline 9 & $(-2) 4.86756$ & $(-2) 8.33773$ & $(-1) 1.22999$ & $(-1) 1.46186$ & $(-1) 1.85038$ & $(-1) 2.22922$ & $(-1) 2.71386$ & $(-1) 3.61978$ & 1763 \\
\hline 10 & $(-2) 6.28902$ & $(-1) 1.02523$ & $(-1) 1.46056$ & $(-1) 1.70965$ & $(-1) 2.12009$ & $(-1) 2.51368$ & $(-1) 3.00969$ & $(-1) 3.91964$ & $(-1) 5.00000$ \\
\hline 12 & $(-2) 9.34092$ & $(-1) 1.41309$ & $(-1) 1.90916$ & $(-1) 2.18338$ & $(-1) 2.62378$ & $(-1) 3.03537$ & $(-1) 3.54216$ & $(-1) 4.44507$ & 8306 \\
\hline 15 & $(-1) 1$ & 97869 & $(-1) 2.53227$ & $(-1) 2.82755$ & .28933 & $(-1) 3$ & 21429 & $(-1)$ & 5494 \\
\hline 20 & $(-1) 2.17852$ & $(-1) 2.82676$ & $(-1) 3.42056$ & $(-1) 3.72565$ & $(-1) 4.18965$ & $(-1) 4.59995$ & $(-1) 5.08035$ & $(-1) 5.88324$ & 249 \\
\hline 24 & $(-1) 2.73505$ & $(-1) 3.41011$ & $(-1) 4.00870$ & $(-1) 4.31029$ & $(-1) 4.76229$ & $(-1) 5.15604$ & $(-1) 5.61078$ & $(-1) 6.35760$ & 4114 \\
\hline 30 & $(-1) 3.46257$ & $(-1) 4.14434$ & $(-1) 4.72890$ & $(-1) 5.01748$ & $(-1) 5.44347$ & $(-1) 5.80880$ & $(-1) 6.22473$ & $(-1) 6.89542$ & 8457 \\
\hline 40 & $(-1) 4.42659$ & $(-1) 5$ & $(-1) 5.62054$ & $(-1) 5.88188$ & $(-1) 6$ & $(-1) 6.58193$ & $(-1) 6.94121$ & $(-1) 7.50951$ & 08076 \\
\hline 60 & $(-1) 5.72440$ & $(-1) 6.28834$ & $(-1) 6.73841$ & $(-1) 6.95108$ & $(-1) 7.25497$ & $(-1) 7.50695$ & $(-1) 7.78512$ & $(-1) 8.21627$ & 967 \\
\hline 120 & $(-1) 7.50435$ & $(-1) 7.87548$ & $(-1) 8.16038$ & $(-1) 8.29183$ & $(-1) 8.47637$ & $(-1) 8.62660$ & $(-1) 8.78968$ & $(-1) 9.03703$ & $(-1) 9.27398$ \\
\hline$\infty$ & 1.00000 & 1.00000 & 1.00000 & 1.00000 & 1.00000 & 1.00000 & 1.00000 & 1.00000 & 1.00000 \\
\hline
\end{tabular}

The numbers in parentheses indicate the power of ten by which the number following is to be multiplied, e.g., $(-1) 1.23456=0.123456$. 
PERCENTAGE POINTS OF THE BETA DISTRIBUTION

$\nu_{1}=12$

\begin{tabular}{|c|c|c|c|c|c|c|c|c|c|}
\hline$\nu_{2}$ & 000 & $p=0.001$ & 005 & 0.01 & $p=0.025$ & 0.05 & $p=0.1$ & $p=0.25$ & $=0.5$ \\
\hline I & $(-9) 1.36463$ & $(-7) 1.36463$ & 41160 & 36469 & $(-5) 8.53133$ & $(-4) 3.41545$ & $(-3) 1.37087$ & $(-3) 8.78142)$ & $(-2) 3.87462$ \\
\hline 1.1 & $(-9) 7.45283$ & $(-7) 4.90347$ & $(-6) 9.14893$ & $(-5) 3.22649$ & $(-4) 1.70786$ & $(-4) 6.03093$ & 3727 & 591( & $(-2) 4.58028$ \\
\hline 1.2 & $(-8) 3.07656$ & $(-6) 1.42802$ & $(-5) 2.08791$ & $(-5) 6.62963$ & $(-4) 3.05525$ & $(-4) 9.72004$ & $(-3) 3.10655$ & $(-2) 1.48370$ & $(-2) 5.29278$ \\
\hline 1.3 & $(-7) 1.02386$ & $(-6) 3.53759$ & $(-5) 4.20821$ & $(-4) 1.22272$ & $(-4) 5.01233$ & $(-3)$ & 809 & 2714 & 0789 \\
\hline 1.4 & $(-7) 2.87640 \mid$ & $(-6) 7.71667$ & $(-5) 7.69214$ & $(-4) 2.07136$ & $(-4) 7.68172$ & $(-3) 2.07573$ & $(-3) 5.64632$ & $(-2) 2.19234)$ & $(-2) 6$ \\
\hline 1.5 & $(-7) 7.05599$ & $(-5) 1.52023$ & $(-4) 1.30020$ & $(-4) 3.27816$ & $6(-3) 1.11479$ & $(-3) 2.82283$ & $(-3) 7.20246$ & $(-2) 2.57594$ & 3451 \\
\hline 1.6 & $(-6) 1.55015$ & $(-5) 2.75680$ & $(-4) 2.06221$ & $(-4) 4.90868$ & $8(-3) 1.54762$ & $(-3) 3.70296$ & $(-3) 8.93584$ & $(-2) 2.97504$ & 4217 \\
\hline 1.7 & $(-6) 3.10969$ & $(-5) 4.66916$ & $(-4) 3.10360$ & $(-4) 7.02229$ & $9(-3) 2.07133$ & $(-3) 4.71523$ & $(-2) 1.08348$ & $(-2) 3.38714$ & $(-2) 8.84433$ \\
\hline 1.8 & $(-6) 5$ & $(-5) 7.47010$ & $(-4) 4.47080$ & $(-4) 9.67069$ & $(-3) 2.68893$ & $(-3) 5.85699$ & $(-2) 1.28875$ & 1011 & 1010 \\
\hline 1.9 & $(-5) 1.00880$ & $(-4) 1.13908$ & $(-4) 6.20691$ & $(-3) 1.28972$ & $(-3) 3.40196$ & $(-3) 7.12432$ & $(-2) 1.50819$ & $(-2) 4.24212$ & 289 \\
\hline 2 & $(-5) 1.66674$ & $(-4) 1.66736$ & $(-4) 8.35075$ & $(-3) 1.67365$ & $5(-3) 4.21074$ & $(-3) 8.51244$ & $(-2) 1.74068$ & $(-2) 4.68157$ & 09101 \\
\hline 2.2 & $(-5) 3.98075$ & $(-4) 3.23109$ & $(-3) 1.39923$ & $(-3) 2.63530$ & $0(-3) 6.11206$ & $(-2) 1.16292$ & $(-2) 2.24046$ & $(-2) 5.57757$ & 22490 \\
\hline 2.5 & $(-4) 1.13936$ & $(-4) 7.19854$ & $(-3) 2.61971$ & $(-3) 4.58111$ & $(-3) 9.64291$ & $(-2) 1.70686$ & $(-2) 3.06271$ & $(-2) 6.94883$ & $(-1) 1.41958$ \\
\hline 3 & $(-4) 4.17656$ & $(-3) 1$ & $(-3)$ & $(-3) 9.15690$ & $d(-2$ & $(-2)$ & $(-2)$ & 59231 & 2751 \\
\hline 3.5 & $(-3) 1.07030$ & $(-3) 4.01103$ & $(-2) 1.01752$ & $(-2) 1.52612$ & $2(-2) 2.62865$ & $(-2) 4.00599$ & $9 .(-2) 6.19773$ & $(-1) 1.15527$ & $(-1)$ \\
\hline 4 & & $(-3) 6.98151$ & $(-2) 1.58443$ & & & $6(-2) 5.33755$ & $(-2) 7.88234$ & $(-1) 1.37974$ & $(-1) 2.28490$ \\
\hline 4.5 & & & $(-2) 2.25733$ & $(-2) 3.11289$ & $9(-2) 4.80279$ & $9(-2) 6.73755$ & $(-2) 9.59337$ & $(-1) 1.59766$ & $(-1) 2.53718$ \\
\hline 5 & & $(-2) 1.55272$ & $(-2) 3.01907$ & $(-2) 4.04337$ & $7(-2) 6.00279$ & $9(-2) 8.17896$ & $(-1) 1.13074$ & $(-1) 1.80817$ & $(-1) 2.77379$ \\
\hline 6 & $(-2) 1.23211$ & $(-2) 2.70434$ & $(-2) 4.74640$ & $(-2) 6.08396$ & $6(-2) 8.52334$ & $4(-1) 1.11113$ & $(-1) 1.46855$ & $(-1) 2$ & $(-1) 3.20519$ \\
\hline 7 & $(-2) 2.06652$ & $(-2) 4.08192$ & 65925 & $(-2) 8.27139$ & $9(-1) 1.11129$ & $9(-1) 1.40291$ & $(-1) 1.79407$ & $(-1) 2.57241$ & $(-1)$ \\
\hline 8 & $(-2) 3.07906$ & $(-2) 5.62059$ & $(-2) 8.67877$ & $(-1) 1.05262$ & $(-1) 1.36996$ & $(-1) 1.68750$ & $(-1) 2.10396$ & $(-1) 2.90986$ & 3085 \\
\hline 9 & $(-2) 4.23330$ & $(-2) 7.26744$ & $(-1) 1.07490$ & $(-1) 1.27955$ & $(-1) 1.62400$ & $(-1) 1.96184$ & $(-1) 2.39702$ & $(-1) 3.22049$ & $(-1) 4.23872$ \\
\hline 10 & $(-2) 5.49568$ & $(-2) 8.98132$ & $(-1) 1.28311$ & $(-1) 1.50443$ & $(-1) 1.87086$ & $(-1) 2.22441$ & $(-1) 2.67318$ & $(-1) 3.50681$ & $(-1) 4$ \\
\hline 12 & $(-2) 8.23433$ & $(-1) 1.2$ & $(-1)$ & $(-1) 1.939$ & $(-1) 2.33$ & $(-1) 2.71250$ & $(-1) 3.17719$ & $(-1) 4.01580$ & $(-1$ \\
\hline 15 & $(-1) 1.25807$ & $(-1) 1.77002$ & $(-1) 2.27283$ & $(-1) 2.54259$ & $(-1) 2.96681$ & $(-1) 3.35542$ & $(-1) 2.82606$ & $(-1) 4.64974$ & $(-1$ \\
\hline 20 & $(-1) 1.97231\}$ & $(-1) 2.56822$ & $(-1) 3.11843$ & $(-1) 3.40290$ & $(-1) 3.83804$ & $(-1) 4.22556$ & $(-1) 4.68292$ & $(-1) 5.45736$ & $(-1)$ \\
\hline 24 & $(-1) 2.50026$ & $(-1) 3.12846$ & $(-1) 3.69009$ & $(-1) 3.97487$ & $(-1) 4.40417$ & $(-1) 4.78082$ & $(-1) 5.21929$ & $(-1) 5.94873$ & $(-1) 6$ \\
\hline 30 & $(-1) 3.20203$ & $(-1) 3.84579$ & $(-1) 4.40239$ & $(-1) 4.67894$ & $(-1) 5.08954$ & $(-1) 5.44418$ & $(-1) 5.85110$ & $(-1) 6.51560$ & $(-1) 7.2$ \\
\hline 40 & $(-1) 4$ & $(-1) 4.77842$ & $(-1) 5.30242$ & $(-1) 5.55726$ & $(-1) 5.92963$ & $(-1) 6.24595$ & $(-1) 6.60341$ & $(-1) 7.17576$ & $(-1) 7$ \\
\hline 60 & $(-1) 5.45942$ & $(-1) 6.01303$ & $(-1) 6.45839$ & $(-1) 6.67012$ & $(-1) 6.97429$ & $(-1) 7.22815$ & $(-1) 7.51041$ & $(-1) 7.95294$ & 39537 \\
\hline 120 & $(-1) 7.30870$ & $(-1) 7.68276$ & $(-1) 7.97198$ & $(-1) 8.10616$ & $(-1) 8.29544$ & $(-1) 8.45045$ & $(-1) 8.61980$ & $(-1) 8.87937)($ & $(-1) 9$. \\
\hline$\infty$ & 1.00000 & 1.00000 & 1.00000 & 1.00000 & 1.00000 & 1.00000 & 1.00000 & 1.00000 & 1.00000 \\
\hline
\end{tabular}

The numbers in parentheses indicate the power of ten by which the number following is to be multiplied, e.g., $(-1) 1.23456=0.123456$. 
PERCENTAGE POINTS OF THE BETA DISTRIBUTION

$v_{1}=15$

\begin{tabular}{|c|c|c|c|c|c|c|c|c|c|}
\hline$\nu_{2}$ & $p=0.0001$ & $p=0.001$ & $p=0.005$ & $p=0.01$ & $p=0.025$ & $p=0.05$ & $p=0.1$ & $p=0.25$ & $p=0.5$ \\
\hline । & $(-9) 1.08267 \mid$ & $(-7) 1.08267$ & $(-6) 2.70670$ & $(-5) 1.08272$ & $(-5) 6.76865$ & $(-4) 2.70984$ & $(-3) 1.08778$ & $(-3) 6.97342$ & $(-2) 3.08671$ \\
\hline I. 1 & $(-9) 5.91806$ & $(-7) 3.89369$ & $(-6) 7.26488$ & $(-5) 2.56206$ & $(-4) 1.35618$ & $(-4) 4.78927$ & $(-3) 1.69751$ & $(-3) 9.26941$ & $(-2) 3$ \\
\hline 1.2 & $(-8) 2.44510$ & $(-6) 1.13492$ & $(-5) 1.65937$ & $(-5) 5.26894$ & $(-4) 2.42824$ & $(-4) 7.72579$ & $(-3)^{2} .46973$ & $(-2) 1.18100$ & 23003 \\
\hline 1.3 & $(-8) 8.14409$ & $(-6) 2.81390$ & $(-5) 3.34734$ & $(-5) 9.72595$ & $(-4) 3.98715$ & $(-3) 1.16169$ & $(-3) 3.40442$ & $(-2) 1.45612$ & $(-2) 4.80921$ \\
\hline 1.4 & $(-7) 2.28990$ & $(-6) 6.14323$ & $(-5) 6.12375$ & $(-4) 1.64904$ & $(-4) 6.11589$ & $(-3) 1.65284$ & $(-3) 4.49764$ & $(-2) 1.74928$ & $(-2) 5.38980$ \\
\hline 1.5 & $(-7) 5.62195$ & $(-5) 1.21126$ & $(-4) 1.03597$ & $(-4) 2.61201$ & $(-4) 8.88325$ & $(-3) 2.24977$ & $(-3) 5.74290$ & $(-2) 2.05788$ & $(-2) 5.96998$ \\
\hline 1.6 & $(-6) 1.23612$ & $(-5) 2.19834$ & $(-4) 1$ & $(-4) 3.91448$ & $(-3) 1.23430$ & $(-3) 2.95394$ & $(-3) 7.13214$ & $(-2) 2.37963$ & $(-2) 6.54840$ \\
\hline 1.7 & $(-6) 2.48177$ & $(-5) 3.72636$ & $(-4) 2.47698$ & $(-4) 5.60471$ & $(-3) 1.65343$ & $(-3) 3.76491$ & $(-3) 8.65653$ & $(-2) 2.71260$ & $(-2) 7.12405$ \\
\hline 1.8 & $(-6) 4.61884$ & $(-5) 5.96658$ & $(-4) 3.57109$ & $(-4) 7.72494$ & $=(-3) 2 \cdot 14829$ & $(-3) 4.68088$ & $(-2) 1.03070$ & $(-2) 3.05510$ & 69619 \\
\hline 1.9 & $(-6) 8.06401$ & $(-5) 9.10548$ & 96189 & $(-3) 1.03109$ & $(-3) 2.72034$ & $(-3) 5.69901$ & 20743 & $(-2) 3.40569$ & $(-2)$ \\
\hline 2 & $(-5) 1.33339$ & $(-4) 1.33391$ & $(-4) 6.68116$ & $(-3) 1.33915$ & $(-3) 3.37002$ & $(-3) 6.81577$ & $(-2) 1.39499$ & $(-2) 3.76313$ & $(-2) 8.82775$ \\
\hline 2.2 & $(-5)^{3}$ & $(-4) 2$ & $(-3) 1$ & $(-3) 2$ & $(-3) 4$ & $(-3) 9.32879$ & $(-2)$ & 9434 & $(-2)$ \\
\hline 2.5 & $(-5) 9.15029$ & $(-4) 5.78156$ & $(-3) 2$ & $(-3) 3.68075$ & $(-3) 7.75157$ & $(-2) 1.37309$ & $(-2) 2.46713$ & 1965 & 5688 \\
\hline 3 & $(-4) 3.36676$ & $(-3) 1.56772$ & $(-3) 4.62057$ & $(-3) 7.38765$ & $(-2) 1.38382$ & $(-2) 2.24647$ & $(-2) 3.70349$ & 53244 & $(-1)$ \\
\hline 3.5 & $(-4) 8.65884$ & $(-3) 3.24587$ & $(-3) 8.23894$ & $(-2) 1.23631$ & $(-2) 2.13170$ & $(-2) 3.25296$ & $(-2) 5.04345$ & $(-2) 9.45166$ & $(-1)$ \\
\hline 4 & $(-3) 1.77807$ & 67040 & $(-2)$ & $(-2) 1.84355$ & $(-2) 2.98846$ & $(-2) 4.35412$ & $(-2) 6.44585$ & 3497 & $(-1)$ \\
\hline 4.5 & $(-3) 3.13959$ & $(-3) 8.83637$ & $(-2) 1.8$ & $(-2) 2.54211$ & $(-2) 3.92830$ & $(-2) 5.52082$ & 88244 & 32108 & $(-1)$ \\
\hline 5 & $(-3) 4.98428$ & $(-2) 1.27012$ & $(-2) 2.47286$ & $(-2) 3.31492$ & $(-2) 4.93019$ & $(-2) 6.73116$ & $(-2) 9.33355$ & $(-1) 1.50258$ & $(-1)^{2} .32879$ \\
\hline 6 & $(-2) 1.01349$ & $(-2) 2.22733$ & $(-2) 3.91620$ & $(-2) 5.02576$ & $(-2) 7.05633$ & $(-2) 9.22072$ & $(-1) 1.22278$ & $(-1) 1.85002$ & $(-1)^{2}$ \\
\hline 7 & $(-2) 1.71029$ & $(-2) 3.38401$ & $(-2) 5$ & $(-2) 6$ & $(-2) 9.26946$ & $(-1) 1.17329$ & $(-1) 1.50591$ & $(-1) 2.17579$ & $(-1) 3.06824$ \\
\hline 8 & $(-2) 2.56325$ & $(-2) 4$ & $(-2) 7$ & $(-2) 8.81767$ & 5078 & 42156 & $(-1)$ & 22 & $1-1$ \\
\hline 9 & $(-2) 3.54388$ & $(-2) 6.09876$ & $(-2) 9.04642$ & $(-1) 1.07873$ & $(-1) 1.37320$ & $(-1) 1.66385$ & $(-1) 2.04108$ & 440 & $(-1)$ \\
\hline 10 & $(-2) 4.62528$ & $(-2) 7.57966$ & $(-1) 1.08625$ & $(-1) 1.27596$ & $(-1) 1.59172$ & $(-1) 1.89842$ & $(-1) 2.2$ & 2969 & 4506 \\
\hline 12 & $(-2) 6.99926$ & $(-1) 1.06536$ & $(-1) 1.44892$ & $(-1) 1.66333$ & $(-1) 2.01146$ & $(-2) 2.34134$ & $(-1) 2.75401$ & $(-1)$ & 41622 \\
\hline 15 & $(-1) 1.08354$ & $(-1) 1.53020$ & $(-1) 1.97247$ & .21132 & $(-1) 2.58927$ & 3820 & $1-$ & $(-$ & $(-1$ \\
\hline 20 & $(-1) 1.72976$ & $(-1) 2.26177$ & 5750 & 1565 & $(-1) 3.41318$ & $(-1) 3.77009$ & 9516 & 2564 & $(-1)$ \\
\hline 24 & $(-1) 2.21877$ & $(-1) 2.78808$ & $(-1) 3.30197$ & $(-1) 3.56448$ & $(-1) 3.96292$ & $(-1) 4.31537$ & $(-1) 4.72942$ & $(-1) 5.42848$ & $(-1) 6.19406$ \\
\hline 30 & $(-1) 2.88245$ & $(-1) 3.47660$ & $(-1) 3.99542$ & $(-1) 4.25516$ & $(-1) 4.64344$ & $(-1) 4.98156$ & $(-1) 5.37306$ & $(-1) 6.02174$ & $(-1) 6.71684$ \\
\hline 40 & $(-1) 3.80293$ & $(-1) 4.39540$ & $(-1) 4.89499$ & $(-1) 5.13979$ & $(-1) 5.49990$ & $(-1) 5.80830$ & $(-1) 6.15990$ & 3084 & $(-1) 7.32847$ \\
\hline 60 & $(-1) 5.11372$ & $(-1) 5.65123$ & $(-1) 6.08785$ & $(-1) 6.29693$ & $(-1) 6.59924$ & $(-1) 6.85350$ & $(-1) 7.13856$ & $(-1) 7.59150$ & $(-1)$ \\
\hline 120 & $(-1) 7.04241$ & $(-1) 7.41889$ & $(-1) 7.71254$ & $(-1) 7.84968$ & $(-1) 8.04425$ & $(-1) 8.20471$ & $(-1) 8.38135$ & $(-1) 8.65539$ & $(-1) 8.92730$ \\
\hline$\infty$ & 1.00000 & 1.00000 & 1.00000 & 1.00000 & 1.00000 & 1.00000 & 1.00000 & 1.00000 & 1.00000 \\
\hline
\end{tabular}

The numbers in parentheses indicate the power of ten by which the number following is to be multiplied, e.g., $(-1) 1.23456=0.123456$. 


\begin{tabular}{|c|c|c|c|c|c|c|c|c|c|}
\hline $7 / 2$ & $p=0.0001$ & $p=0.001$ & $p=0.005$ & $p=0.01$ & $p=0.025$ & $p=0.05$ & $p=0.1$ & $p=0.25$ & $p=0.5$ \\
\hline I & $(-10) 8.05272$ & $(-8) 8.05272$ & $(-6) 2.01320$ & $(-6) 8.05311$ & $(-5) 5.03447$ & $(-4) 2.01562$ & $(-4) 8.09189$ & $(-3) 5.19143$ & .30514 \\
\hline 1.1 & $(-9) 4.40556$ & $(-7) 2.89857$ & $(-6) 5.40818$ & $(-5) 1.90727$ & $7(-4) 1.00959$ & $(-4) 3.56548$ & $(-3) 1.26395$ & $(-3) 6.90868$ & $(-2) 2.73370$ \\
\hline 1.2 & $(-8) 1.82176$ & $(-7) 8.45588$ & $(-5) 1.23634$ & $(-5) 3.92572$ & $(-4) 1.80925$ & $(-4) 5.75678$ & $(-3) 1.84069$ & $(-3) 8.81261$ & $(-2) 3.16908$ \\
\hline 1.3 & $(-8) 6.07301$ & $(-6) 2.09831$ & $(-5) 2.49611$ & $(-5) 7.25268$ & $(-4) 2.97335$ & $(-4) 8.66396$ & $(-3) 2.53977$ & $(-2) 1.08786$ & $(-2) 3.60874$ \\
\hline 1.4 & $(-7) 1.70900$ & $(-6) 4.58484$ & $(-5) 4.57033$ & $(-4) 1.23074$ & $(-4) 4.56478$ & $(-3) 1.23381$ & $(-3) 3.35862$ & $(-2) 1.30846$ & $(-2) 4.05083$ \\
\hline 1.5 & $(-7) 4.19929$ & $(-6) 9.04750$ & $(-5) 7.73823$ & $(-4) 1.95109$ & $(-4) 6.63606$ & $(-3) 1.68094$ & $=(-3) 4.29277$ & $(-2) 1.54118$ & $(-2) 4.49397$ \\
\hline 1.6 & $(-7) 9.24084$ & $(-5) 1.64341$ & $(-4) 1.22939$ & $(-4) 2.92647$ & $(-4) 9.22863$ & $(-3) 2.20909$ & $(-3) 5.33657$ & $(-2) 1.78435$ & $(-2) 4.93712$ \\
\hline 1.7 & $(-6) 1.85681$ & $(-5) 2.78800$ & $(-4) 1.85329$ & $(-4) 4.19363$ & $(-3) 1.23732$ & $(-3) 2.81817$ & $(-3) 6.48375$ & $(-2) 2.03654$ & $(-2) 5.37951$ \\
\hline 1.8 & $(-6) 3.45854$ & $(-5) 4.46775$ & $(-4) 2.67412$ & $(-4) 5.78493$ & $(-3) 1.60906$ & $(-3) 3.50706$ & $(-3) 7.72781$ & $(-2) 2.29654$ & $(-2) 5.82052$ \\
\hline 1.9 & $(-6) 6.04316$ & $(-5) 6.82370$ & $(-4) 3.71866$ & $(-4) 7.72793$ & $(-3) 2.03931$ & $(-3) 4.27388$ & $(-3) 9.06225$ & $(-2) 2.56328$ & $(-2) 6.25971$ \\
\hline 2 & $(-5) 1.00005$ & $(-4) 1.00045$ & $(-4) 5.01129$ & $(-3) 1.00453$ & $(-3) 2.52858$ & $(-3) 5.11620$ & $(-2) 1.04807$ & $(-2) 2.83583$ & $(-2) 6.69670$ \\
\hline 2.2 & $(-5) 2.39603$ & $(-4) 1.94491$ & $(-4) 8.42428$ & $(-3) 1.58702$ & $(1-3) 3.68332$ & $(-3) 7.01588$ & $(-2) 1.35460$ & $(-2) 3.39532$ & $(-2)$ \\
\hline 2.5 & $(-5) 6.88983$ & $(-4) 4.35357$ & $(-3) 1.58496$ & $(-3) 2.77270$ & $(-3) 5.84216$ & $(-2) 1.03563$ & $(-2) 1$ & $(-2)$ & $(-2)$ \\
\hline 3 & $(-4) 2.54476$ & $(-3) 1.18514$ & $(-3) 3.49426$ & $(-3) 5.58872$ & $(-2) 1.04768$ & $(-2) 1.70257$ & $(-2) 2.81189$ & $(-2) 5.74670$ & 09077 \\
\hline 3.5 & $(-4) 6.56918$ & $(-3) 2.46324$ & $(-3) 6.25613$ & $(-3) 9.39237$ & $(-2) 1.62122$ & $(-2) 2.47734$ & $(-2) 3.84938$ & 25395 & $(-1)$ \\
\hline 4 & $(-3) 1.35389$ & $(-3) 4.31963$ & $(-3) 9.81967$ & $(-2) 1.40651$ & $(-2) 2.28312$ & $(-2) 3.33192$ & $(-2)$ & $(-2) 8.76100$ & $(-1)$ \\
\hline 4.5 & $(-3) 2.39916$ & $(-3) 6.75690$ & $(-2) 1.41008$ & $(-2) 1.94764$ & $(-2) 3.01461$ & $(-2) 4.24479$ & $(-2) 6$ & $(-1) 1.02545$ & $(-1) 1$ \\
\hline 5 & $(-3) 3.82223$ & $(-3) 9.74863$ & $(-2) 1.90064$ & $(-2) 2.55033$ & $(-2) 3.80023$ & $(-2) 5.19953$ & $(-2) 7.23244$ & $(-1) 1.17262$ & $(-1) 1.83736$ \\
\hline 6 & $(-3) 7.82558$ & $(-2) 1.72215$ & $(-2) 3.03374$ & $(-2) 3.89821$ & $(-2) 5.48606$ & $(-2) 7$ & $(-2) c$ & $(-1) 1$ & $(-1) 2,16686$ \\
\hline 7 & $(-2) 1.32941$ & $(-2) 2.63522$ & $(-2) 4.31888$ & $(-2) 5.38011$ & $(-2) 7.26633$ & $(-2) 9.22382$ & $(-1) 1.18856$ & $(-1) 1.73162$ & $(-1) 2.47110$ \\
\hline 8 & $(-2) 2.00532$ & $(-2) 3.67658$ & $(-2) 5.70760$ & $(-2) 6.94554$ & $(-2) 9.09204$ & $(-1) 1.12666$ & $(-1) 1.41611$ & $(-1) 1.99127$ & $(-1) 2.75276$ \\
\hline 9 & $(-2) 2.78984$ & $(-2) 4.81406$ & $(-2) 7.16351$ & $(-2) 8.55840$ & $(-1) 1.09305$ & $(-1) 1.32882$ & $(-1) 1.63736$ & $(-1) 2.23758$ & $(-1) 3.01421$ \\
\hline 10 & $(-2) 3.66313$ & $(-2) 6.02138$ & $(-2) 8.65948$ & $(-1) 1.01929$ & $(-1) 1.27598$ & $(-1) 1.52718$ & $(-1) 1.85134$ & $(-1)^{2} .47100$ & $(-1) 3.25751$ \\
\hline 12 & $(-2) 5.60672$ & $(-2) 8.56555$ & $(-1) 1.16961$ & $(-1) 1.34576$ & $(-1) 1.63364$ & $(-1) 1.90865$ & $(-1) 2.25591$ & $(-1) 2.90170$ & $(-1) 3.69670$ \\
\hline 15 & $(-2) 8.81504$ & $(-1) 1.25033$ & $(-1) 1.61897$ & $(-1) 1.81955$ & $(-1) 2.13925$ & $(-1) 2.43702$ & $(-1) 2.80446$ & $(-1) 3.46790$ & $(-1) 4.25790$ \\
\hline 20 & $(-1) 1.43852$ & $(-1) 1.89037$ & $(-1) 2.31600$ & $(-1) 2.53953$ & $(-1) 2.88643$ & $(-1) 3.20087$ & $(-1) 3.57930$ & $(-1) 4.24087$ & $(-1) 5.00000$ \\
\hline 24 & $(-1) 1.87255$ & $(-1) 2.36535$ & $(-1) 2.81533$ & $(-1) 3.04723$ & $(-1) 3.40206$ & $(-1) 3.71901$ & $(-1) 4.09535$ & $(-1) 4.74178$ & $(-1) 5.46857$ \\
\hline 30 & $(1) 2.47770$ & $(-1) 3.00434$ & $(-1) 3.46978$ & $(-1) 3.70493$ & $(-1) 4.05936$ & $(-1) 4.37107$ & $(-1) 4.73588$ & $(-1) 5.35080$ & $(-1) 6.02708$ \\
\hline 40 & $(-1) 3.34605$ & $(-1) 3.88728$ & $(-1) 4.34932$ & $(-1) 4.57784$ & $(-1) 4.91677$ & $(-1) 5.20988$ & $(-1) 5.54762$ & $(-1) 6.10536$ & $(-1) 6.70415$ \\
\hline 60 & $(-1) 4.63816$ & $(-1) 5.14884$ & $(-1) 5.56875$ & $(-1) 5.77167$ & $(-1) 6.06738$ & $(-1) 6.31847$ & $(-1) 6.60286$ & $(-1) 7.06202$ & $(-1) 7.54199$ \\
\hline 120 & $(-1) 6.65337$ & $(-1) 7.03036$ & $(-1) 7.32770$ & $(-1) 7.46771$ & $(-1) 7.66781$ & $(-1) 7.83425$ & $(-1) 8.01917$ & $(-1) 8.31026$ & $(-1) 8.60551$ \\
\hline$\infty$ & 1.00000 & 1.00000 & 0000 & 1.00000 & 1.00000 & 1.00000 & 1.00000 & 1.00000 & 1.00000 \\
\hline
\end{tabular}


PERCENTAGE POINTS OF THE BETA DISTRIBUTION

$\nu_{1}=24$

\begin{tabular}{l|l|l|l|l|l|l|l|l|l}
$\nu_{2}$ & $p=0.0001$ & $p=0.001$ & $p=0.005$ & $p=0.01$ & $p=0.025$ & $p=0.05$ & $p=0.1$ & $p=0.25$ & $p=0.5$ \\
\hline
\end{tabular}

$1 \quad(-10) 6.68273(-8) 6.68273(-6) 1.67070(-6) 6.68306)(-5) 4.17799(-4) 1.67273(-4) 6.71570|(-3) 4.31014|(-2) 1.91679)$

$1.1 \mid(-9) 3.65762)(-7) 2.40647(-6) 4.49002(-5) 1.58347)(-5) 8.38199(-4) 2.96025)(-3) 1.04948)(-3) 5.73917)(-2) 2.27495$

$1.2(-8) 1.51311)(-7) 7.02329(-5) 1.02688(-5) 3.26064)(-4) 1.50275(-4) 4.78170(-3) 1.52908)(-3) 7.32509(-2) 2.63937)$

$1.3(-8) 5.04625(-6) 1.74355(-5) 2.07410(-5) 6.02651)(-4) 2.47071)(-4) 7.19968(-3) 2.11083)(-3) 9.04772(-2) 3.00794$

$1.4(-7) 1.42066(-6) 3.81128(-5) 3.79923(-4) 1.02310(-4) 3.79476)(-3) 1.02575(-3) 2.79275)(-2) 1.08891(-2) 3.37912$

$1.5(-7) 3.49224(-6) 7.52415(-5) 6.43536(-4) 1.62261)(-4) 5.51903(-3) 1.39811(-3) 3.57128(-2) 1.28337(-2) 3.75175$

$1.6(-7) 7.68812(-5) 1.36727(-4) 1.02283(-4) 2.43480(-4) 7.67856)(-3) 1.83824(-3) 4.44187(-2) 1.48677)(-2) 4.12497)$

$1.7(-6) 1.54545(-5) 2.32050(-4) 1.54254(-4) 3.49054(-3) 1.02995(-3) 2.34616(-3) 5.39947(-2) 1.69797(-2) 4.49812$

$1.8(-6) 2.87978(-5) 3.72012(-4) 2.22667(-4) 4.81709(-3) 1.33997(-3) 2.92104(-3) 6.43879)(-2) 1.91594(-2) 4.87069$

$1.9(-6) 5.03393(-5) 5.68415(-4) 3.09772(-4) 6.43775(-3) 1.69903(-3) 3.56140(-3) 7.55455(-2) 2.13982(-2) 5.24227$

$2(-6) 8.33372(-5) 8.33716(-4) 4.17625(-4) 8.37177(-3) 2.10759(-3) 4.26532(-3) 8.74161(-2) 2.36884(-2) 5.61257$

$2.2(-5) 1.99830(-4) 1.62209(-4) 7.02637(-3) 1.32375(-3) 3.07284(-3) 5.85468(-2) 1.13101(-2) 2.83978(-2) 6.34838$

$2.5(-5) 5.75300(-4) 3.63533(-3) 1.32360(-3) 2.31571)(-3) 4.88052(-3) 8.65484(-2) 1.55827(-2) 3.57082(-2) 7.43782$

$3(-4) 2.12902(-4) 9.91592(-3) 2.92417(-3) 4.67770(-3) 8.77246(-2) 1.42637(-2) 2.35788(-2) 4.83067(-2) 9.20988$

$3.5(-4) 5.50648(-3) 2.06506(-3) 5.24642(-3) 7.87848(-2) 1.36066(-2) 2.08063(-2) 3.23658(-2) 6.11648(-1) 1.09238$

$4(-3) 1.13701)(-3) 3.62852(-3) 8.25219(-2) 1.18239(-2) 1.92067|(-2) 2.80534|(-2) 4.16910)(-2) 7.40950(-1) 1.25791$

$4.5(-3) 2.01859(-3) 5.68701(-2) 1.18749(-2) 1.64089(-2) 2.54196(-2) 3.58280(-2) 5.13768)(-2) 8.69814(-1) 1.41770)$

$5(-3) 3.22184(-3) 8.22111(-2) 1.60398(-2) 2.15336(-2) 3.21186(-2) 4.39941)(-2) 6.12951)(-2) 9.97493(-1) 1.57195$

$6 \quad(-3) 6.62033(-2) 1.45795(-2) 2.57091(-2) 3.30569(-2) 4.65793(-2) 6.11029(-2) 8.14772(-1) 1.24750(-1) 1.86474$

$7 \quad(-2) 1.12867(-2) 2.23948(-2) 3.67494(-2) 4.58164(-2) 6.19689(-2) 7.87828(-1) 1.01733(-1) 1.48875(-1) 2.13815)$

$8(-2) 1.70846(-2) 3.13614(-2) 4.87595(-2) 5.93902)(-2) 7.78715(-2) 9.66583(-1) 1.21769)(-1) 1.72032)(-1) 2.39393$

$9 \quad(-2) 2.38495(-2) 4.12139(-2) 6.14334(-2) 7.34718(-2) 9.40039(-1) 1.14487(-1) 1.41412)(-1) 1.94198(-1) 2.63369$

$10 \quad(-2) 3.14190(-2) 5.17324(-2) 7.45397(-2) 8.78377(-1) 1.10170(-1) 1.32111)(-1) 1.60559 \mid(-1) 2.15383(-1) 2.85886$

$12(-2) 4.83961)(-2) 7.40878(-1) 1.01390(-1) 1.16809(-1) 1.42097(-1) 1.66363(-1) 1.97161)(-1) 2.54934(-1) 3.27038$

$15 \quad(-2) 7.67648(-1) 1.09153(-1) 1.41696(-1) 1.59478(-1) 1.87934(-1) 2.14568(-1) 2.47618(-1) 3.07845(-1) 3.80594)$

$20 \quad(-1) 1.26899(-1) 1.67247(-1) 2.05489(-1) 2.25671)(-1) 2.57131)(-1) 2.85800(-1) 3.20513(-1) 3.81786(-1) 4.53143$

$24(-1) 1.66661(-1) 2.11176(-1) 2.52096(-1) 2.73293(-1) 3.05878(-1) 3.35148(-1) 3.70116(-1) 4.30776(-1) 5.00000$

$30 \quad(-1) 2.23034(-1) 2.71314(-1) 3.14290(-1) 3.36119(-1) 3.69180(-1) 3.98424(-1) 4.32865(-1) 4.91497(-1) 5.56947$

$40 \quad(-1) 3.05703(-1) 3.56293(-1) 3.99804(-1) 4.21443(-1) 4.53696(-1) 4.81751)(-1) 5.14280(-1) 5.68530(-1) 6.27635$

$60 \quad(-1) 4.32344(-1) 4.81347(-1) 5.21943)(-1) 5.41669(-1) 5.70555(-1) 5.95222(-1) 6.23330(-1) 6.69145(-1) 7.17715$

$120 \quad(-1) 6.37971(-1) 6.75503(-1) 7.05314)(-1) 7.19423(-1) 7.39675(-1) 7.56609(-1) 7.75527(-1) 8.05567(-1) 8.36428$

\begin{tabular}{lll|l|l|l|l|l|l|l|l}
$\infty$ & 1.00000 & 1.00000 & 1.00000 & 1.00000 & 1.00000 & 1.00000 & 1.00000 & 1.00000 & 1.00000 \\
\hline
\end{tabular}


$v_{1}=30$

\begin{tabular}{|c|c|c|c|c|c|c|c|c|c|}
\hline$\nu_{2}$ & $p=0.0001$ & $p=0.001$ & $p=0.005$ & $p=0.01$ & $p=0.025$ & $p=0.05$ & $p=0.1$ & $p=0.25$ & $p=0.5$ \\
\hline 1 & $(-10) 5$ & .32397 & $(-6) 1.33101$ & $(-6) 5.32423$ & $(-5) 3.32851$ & $(-4) 1.33265$ & $(-4) 5.35060$ & $(-3)$ & $(-2)$ \\
\hline 1.1 & $(-9) 2.91517$ & $(-7) 1.91799$ & $(-6) 3.57862$ & $(-5) 1.26205$ & $(-5) 6.68063$ & $(-4) 2.35943$ & $(-4) 8.36542$ & $(-3) 4$ & $(-2) 1$ \\
\hline 1.2 & $(-8) 1.20649$ & $(-7) 5.60004$ & $(-6) 8.18785$ & $(-5) 2.59989$ & $(-4) 1.19824$ & $4(-4) 3$ & $(-3) 1$ & $(-3)$ & $(-2)^{2}$ \\
\hline 1.3 & $(-8) 4.02534$ & $(-6) 1.39081$ & $(-5) 1.65449$ & $(-5) 4.80731$ & $(-4) 1.97091$ & $(-4) 5.74353$ & $(-3) 1.68414$ & $(-3) 7$ & $(-2) 2.40682$ \\
\hline 1.4 & $(-7) 1.13372$ & $(-6) 3.04150$ & $(-5) 3.03189$ & $(-5) 8,16469$ & $(-4) 3.02842$ & $(-4) 8.18656$ & $(-3) 2.22931$ & 341 & $(-2) 2$ \\
\hline 1.5 & $(-7) 2.78806$ & $(-6) 6.00696$ & $(-5) 5.13774$ & $(-4) 1.29544$ & $(-4) 4.40640$ & $(-3) 1.11635$ & $(-3) 2.85219$ & $(-2) 1$ & $(-2) 3$ \\
\hline 1.6 & $(-7) 6.14041$ & $(-5) 1.09202$ & $(-5) 8,16928$ & $(-4) 1.94470$ & $(-4) 6.13325$ & $(-3) 1.46845$ & $(-3) 3.54927$ & $(-2)$ & $(-2) 3$ \\
\hline 1.7 & $(-6) 1.23484$ & $(-5) 1.85412$ & $(-4) 1.23254$ & $(-4) 2$ & $(-4) 8$ & $(-3)$ & $(-3)$ & $(-2$ & $(-2)$ \\
\hline 1.8 & $(-6) 2.30194$ & $(-5) 2.97367$ & $(-4) 1.77992$ & $(-4) 3.85071 \mid$ & $(-3) 1.07125$ & $5 \mid(-3)^{2}$ & $(-3) 5.15016$ & $(-2)$ & $(-2)$ \\
\hline 1.9 & $(-6) 4.02550$ & $(-5) 4.54549$ & $(-4) 2.47725$ & $(-4) 5.14844$ & $(-3) 1.35890$ & $(-3)^{2}$ & $(-3)$ & 86 & $(-2)$ \\
\hline 2 & $(-6) 6.66698$ & $(-5) 6.66978$ & $(-4) 3.34114$ & $(-4) 6.69798$ & $(-3) 1.68643$ & 41371 & $(-3) 6$ & $(-2)$ & $(-2)$ \\
\hline 2.2 & $(-5) 1$ & $(-4) 1.29874$ & $(-4) 5.62604$ & $(-3) 1$ & $(-3)$ & $(-3)$ & $(-3)$ & $(-2)$ & $(-2$ \\
\hline 2.5 & $(-5) 4.61167$ & $(-4) 2.91421$ & $(-3) 1.06115$ & $(-3) 1.85672$ & $(-3)$ & $(-3) 6$ & $(-2)$ & $(-2$ & $(-2$ \\
\hline 3 & $(-4) 1.71002$ & $(-4) 7.96504$ & 34930 & $(-3) 3.75876$ & $(-3)$ & $(-2)$ & $(-2)$ & $(-2)$ & $(-2)$ \\
\hline 3.5 & $(-4) 4.43140$ & $(-3) 1.66212$ & .22404 & $(-3)$ & & & & & \\
\hline 4 & $(-4) 9.16784$ & $(-3) 2$ & $(-3) 6$ & 4358 & $(-2)$ & $(-2)$ & $(-2)^{3}$ & $(-2$ & $(-1)$ \\
\hline 4.5 & $(-3) 1.63073$ & $(-3) 4$ & $(-3) 9.60220$ & $(-2) 1.32742$ & $(-2)$ & $4(-2)^{2}$ & $(-2)$ & $(-2$ & $(-1)$ \\
\hline 5 & $(-3) 2.60773$ & $(-3) 6.65723$ & $(-2) 1.29982$ & $(-2) 1.74593$ & $(-2) 2.60680$ & $(-2)$ & $(-2)$ & $1-2$ & $(-1)$ \\
\hline 6 & $(-3) 5.37867)$ & $(-2) 1.18538$ & $(-2) 2.09243$ & $(-2) 2.69232$ & $(-2)$ & $(-2) 4$ & $(-2) 6$ & $(-1)$ & $(-1)$ \\
\hline 7 & $(-3) 9.20412$ & $(-2)$ & $(-2) 3.00384$ & 4812 & 718 & $(-2) 6$ & $(-2) \varepsilon$ & 12 & \\
\hline 8 & $(-2) 1.39835$ & $(-2) 2.57019$ & $(-2) 4.00235$ & $(-2) 4$ & 920 & $(-2) .7 .96954$ & $(-1) 1.00641$ & $1-$ & $(-$ \\
\hline 9 & $(-2) 1.95914$ & $(-2) 3.39077$ & $(-2) 5.06347$ & $(-2) 6.06234$ & 77119 & $9(-2) 9$ & 7430 & 106 & $(-1$ \\
\hline 10 & $(-2) 2.59013$ & $(-2) 4.27234$ & 16840 & $(-2) 7.27760$ & 14658 & 99906 & $(-1)$ & 6642 & $(-1$ \\
\hline 12 & $(-2) 4.01728$ & $(-2) 6.16348$ & 45501 & 5422 & $(-1)$ & $(-1) 1$ & $(-1) 1$ & $(-1$ & \\
\hline 15 & $(-2) 6.43439$ & $(-2)$ & 19424 & 622 & 46 & $\mid(-1$ & $(-1$ & $1-$ & \\
\hline 20 & $(-1) 1.07924$ & $(-1)$ & $(-1)$ & $(-1)$ & $(-1)$ & $7(-1)$ & $(-1)$ & $(-1$ & $(-$ \\
\hline 24 & 3198 & 2089 & 8108 & 36874 & 871 & 2082 & $(-1) 3$ & 05 & $1-$ \\
\hline 30 & $(-1) 1.94205$ & $(-1) 2$ & 651 & 5337 & $(-1)$ & 005 & 3659 & 8152 & $(-1)$ \\
\hline 40 & $(-1) 2$ & $(-1) 3$ & $(-1)$ & 998 & $(-1)$ & $9(-1)$ & $(-1)$ & $(-1)$ & $(-1)$ \\
\hline 60 & $(-1) 3.92975$ & $(-1) 4.3$ & $(-1) 4.77619$ & $(-1) 4.96465$ & $(-1) 5.24219$ & $(-1) 5.48074$ & $(-1) 5$ & $(-1$ & \\
\hline 120 & $(-1) 6.01685$ & $(-1) 6.3$ & $(-1) 6.68455$ & $(-1) 6.82593$ & $(-1) 7.02995$ & $(-1) 7$ & $(-1) 7.39456$ & $(-1) 7$ & $(-1)$ \\
\hline$\infty$ & 1.00000 & 1.00000 & 1.00000 & 1.00000 & 1.00000 & 1.00000 & 1.00000 & 1.00000 & 1.00000 \\
\hline
\end{tabular}




\section{PERCENTAGE POINTS OF THE BETA DISTRIBUTION}

$\nu_{1}=40$

\begin{tabular}{|c|c|c|c|c|c|c|c|c|c|}
\hline$\nu_{2}$ & OOI & $p=0.001$ & $p=0.005$ & $p=0.01$ & $p=0.025$ & $p=0.05$ & $p=0.1$ & $p=0.25$ & $p=0.5$ \\
\hline I & $(-10$ & 638 & 108 & 7658 & 8602 & $(-5) 9.95349$ & $(-4)$ & 6689 & $(-2$ \\
\hline 1.1 & $(-9)^{2}$ & 3312 & $(-6) 2.67394$ & $(-6) 9.43007$ & $(-5) 4.99181$ & $(-4) 1.76302$ & $(-4) 6.25130$ & 42184 & $(-2) 1.36113$ \\
\hline 1.2 & 01866 & $(-7) 4$ & 2055 & $(-5) 1.94346$ & $(-5) 8.95716$ & $(-4) 2.85033$ & $(-4) 9.11665$ & 37251 & 8168 \\
\hline 1.3 & $(-8) 3$ & 4009 & $(-5) 1.23728$ & $(-5) 3.59507$ & $(-4) 1.47394$ & $(-4) 4$ & $(-3) 1$ & 40722 & $(-2)$ \\
\hline 1.4 & $(-8) 8.48183$ & $(-6) 2.27547$ & $(-5) 2.26829$ & $(-5) 6.10842$ & $(-4) 2.26578$ & $(-4) 6.12535$ & $(-3) 1.66831$ & $(-3) 6.51558$ & 146 \\
\hline 1.5 & 3673 & $(-6) 4.49594$ & $(-5) 3.84539$ & $(-5) 9.69594$ & $=(-4) 3.29818$ & $(-4) 8.35656$ & $(-3) 2 \cdot 13550$ & $(-3) 7.68852$ & $(-2)$ \\
\hline 1.6 & $(-7) 4$ & $(-6) 8.17671$ & $(-5) 6.11694$ & $(-4) 1.45616$ & $(-4) 4.59272$ & $(-3) 1.09973$ & $(-3) 2.65876$ & $(-3) 8.91818$ & 18776 \\
\hline 1.7 & $(-7) 9$ & $(-5) 1.38888$ & $(-5) 9.23280$ & $(-4) 2.08933$ & $(-4) 6.16576$ & $(-3) 1.40490$ & $(-3) 3.23524$ & $(-2) 1.01978$ & $(-2) 2.71712$ \\
\hline 1.8 & $(-6) 1.72504$ & 2844 & $(-4) 1.33388$ & $(-4) 2.88581$ & $(-4) 8.02884$ & $(-3) 1.75078$ & 36195 & 15214 & $(-2)$ \\
\hline 1.9 & $(-6) 3.01789$ & 40774 & $(-4) 1.85723$ & $(-4) 3.86000$ & $(-3) 1.01893$ & $(-3) 2.13662$ & 53591 & 28840 & $(-2)$ \\
\hline 2 & $(-6) 5.00024$ & $(-5) 5.00238$ & $(-4) 2.50596$ & $(-4) 5.02391$ & $(-3) 1.26509$ & $(-3)^{2} .56138$ & $(-3) 5.25417$ & $(-2) 1.42811)$ & $(-2)$ \\
\hline 2.2 & 20093 & $(-5)$ & $(-4) 4.22326$ & $(-4) 7.95750$ & $(-3) 1.84783$ & $(-3) 3.52263$ & $(-3) 6.81249$ & $(-2) 1.71642$ & $(-2)$ \\
\hline 2.5 & $(-5)$ & 017 & $(-4)$ & $(-3) 1.3$ & 94299 & $(-3) 5.22286$ & $(-3) 9.41659$ & 16663 & $(-2) 4.54918$ \\
\hline 3 & $(-4) 1.28769$ & 837 & 76957 & $(-3) 2$ & 31481 & $(-3) \varepsilon$ & 3274 & 4999 & $(-2)$ \\
\hline 3.5 & $(-4) 3.34360$ & $(-3) 1.25430$ & $(-3) 3.18861$ & $(-3) 4.79076$ & 28324 & 842 & 7766 & 5916 & $(-2$ \\
\hline 4 & $(-4)$ & 21295 & $(-3) 5.03734$ & $(-3) 7.22263$ & $(-2) 1.17493$ & $(-2) 1.71910$ & $(-2) 2.56173$ & $(-2) 4.58268$ & 86438 \\
\hline 4.5 & $(-3) 1.23528$ & $(-3) 3.48262 \mid$ & $(-3) 7.28065$ & $(-2) 1.00694$ & $(-2) 1.56262$ & $(-2) 2.20695$ & $(-2) 3.17448$ & $(-2) 5.41321$ & 92470 \\
\hline 5 & $(-3)$ & 5520 & $(-3) 9.87773$ & $(-2) 1.32750$ & $(-2) 1.98411$ & $(-2) 2.72403$ & $(-2) 3.80828$ & 24579 & 6223 \\
\hline 6 & $(-3) 4$ & 3911 & 9730 & 05670 & $(-2) 2.90559$ & $(-2) 3.82235$ & $(-2) 5.11741$ & 90426 & $(-1)$ \\
\hline 7 & $(-3) 7.04073$ & $(-2) 1.39989)$ & $(-2) 2.30341$ & $(-2) 2.87666$ & $(-2) 3.90287$ & 97805 & 734 & 4047 & $(-1)$ \\
\hline 8 & $(-2) 1.07385$ & 97641 & $(-2) 3.08283$ & $(-2) 3.76250$ & $(-2) 4.95076$ & $(-2) 6.16755$ & $(-2) 7.80835$ & $(-1) 1.11449$ & 7343 \\
\hline 9 & 51032 & 1825 & $(-2) 3.91742$ & $(-2) 4.69567 \mid$ & $(-2) 6.03141$ & $(-2) 7.37479 \mid$ & $(-2) 9.15767$ & $(-1) 1.27123$ & $(-1)$ \\
\hline 10 & $(-2)$ & $(-2)$ & $(-2) 4$ & $(-2) 5$ & $(-2) 7$ & $(-2) 8$ & $=(-1) 1$ & $(-1) 1.42398$ & $(-1) 1$ \\
\hline 12 & 25 & 711 & $(-2)$ & $(-2$ & $(-2$ & $(-1$ & $(-1$ & 1711 & 91 \\
\hline 15 & $(-2) 5.07123$ & $(-2) 7.25208$ & $(-2) 9.46972$ & 06932 & $(-1) 1.26689$ & $(-1$ & 889 & 612 & 153 \\
\hline 20 & $(-2)$ & 772 & 1984 & 56507 & $(-1) 1$ & 0496 & $(-1) 2.26415$ & 73207 & $(-1\rangle$ \\
\hline 24 & $(-1)$ & $(-1)$ & $(-1) 1.78286$ & $(-1) 1$ & $(-1) 2.1850 \varphi$ & $(-1) 2.40775$ & $(-1) 2.67777$ & $(-1) 3,15717$ & $(-1) 3.72365$ \\
\hline 30 & $(-$ & $(-1$ & $(-1)$ & $(-1$ & $(-1)$ & $(-1)$ & $(-1) 3.22827$ & $(-1) 3.71223$ & $(-1) 4.27196$ \\
\hline 40 & 288 & $(-1)^{2}$ & $(-1) 3$ & 21106 & 47802 & $(-1) 3.71361$ & $(-1) 3.99102$ & 16497 & $(-1) 5$ \\
\hline 60 & $(-1) 3.41920$ & $(-1) 3.83772$ & $(-1) 4.19127$ & $(-1) 4.36552$ & $(-1) 4.62385$ & $(-1) 4.84767$ & $(-1) 5.10666$ & $(-1) 5$ & $(-1)$ \\
\hline 120 & 年 & $(-1)$ & $(-1) 6$ & $(-1)$ & $(-1$ & $(-1$ & $(-1)$ & 18522 & $(-1) 7.52092$ \\
\hline$\infty$ & $1.0000 \mathrm{~d}$ & 1.00000 & $1.0000 \mathrm{q}$ & 1.00000 & $1.0000 q$ & 1.00000 & 1.00000 & 1.00000 & 1.00000 \\
\hline
\end{tabular}

The numbers in parentheses indicate the power of ten by which the number following is to be multiplied, e.g. , $(-1) 1.23456=0.123456$. 


\begin{tabular}{|c|c|c|c|c|c|c|c|c|c|}
\hline$\nu_{2}$ & $p=0.0001$ & $p=0.001$ & .005 & $p=0.01$ & $p=0.025$ & $p=0.05$ & $p=0.1$ & $p=0.25$ & $p=0.5$ \\
\hline 1 & $(-10) 2.63990$ & $(-8) 2.63990$ & $(-7) 6.59984$ & $(-6) 2.64004$ & $(-5) 1.65046)$ & $(-5) 6.60819$ & $(-4) 2.65347$ & $(-3) 1.70489$ & $(-3) 7.61653$ \\
\hline 1.1 & $(-9) 1.44672$ & $(-8) 9.51846$ & $(-6) 1.77597$ & $(-6) 6.26323$ & $(-5) 3.31546$ & $(-4) 1.17099$ & $(-4) 4.15240$ & $(-3) 2.27401$ & $(-3) 9.06116$ \\
\hline 1.2 & $(-9) 5.99249$ & $(-7) 2.78148$ & $(-6) 4.06683$ & $(-5) 1.29135$ & $(-5) 5.95172$ & $(-4) 1.89400$ & $(-4) 6.05853$ & $(-3) 2.90748$ & $(-2) 1.05377$ \\
\hline 1.3 & $(-8) 2.00102$ & $(-7) 6.91384$ & $(-6) 8.22460$ & $(-5) 2.38978$ & $(-5) 9.79802$ & $(-4) 2.85556$ & $(-4) 8.37556$ & $(-3) 3.59763$ & $(-2) 1.20380$ \\
\hline 1.4 & $(-8) 5.64052$ & $(-6) 1.51322$ & $(-5) 1.50845$ & $(-5) 4.06221$ & $(-4) 1.50683$ & $\mid(-4) 4.07385$ & $(-3) 1.10976$ & $(-3) 4.33769$ & $(-2) 1.35559$ \\
\hline 1.5 & $(-7) 1.38828$ & $(-6) 2.99110$ & $(-5) 2.55832$ & $(-5) 6.45071$ & $(-4) 2.19436$ & $(-4) 5.56031$ & $(-3) 1.42124$ & $(-3) 5.12171$ & $(-2) 1.50869$ \\
\hline 1.6 & $(-7) 3$ & $(-6) 5.44215$ & $(-5) 4.07127$ & $(-5) 9.69193$ & $(-4) 3.05699$ & $(-4) 7.32078$ & $(-3) 1.77037$ & $(-3) 5.94454$ & $(-2) 1.66275$ \\
\hline 1.7 & $(-7)$ & $(-6) 9.24779$ & $(-5) 6.14768$ & $(-4) 1.39121$ & $(-4) 4.10585$ & $(-4) 9.35661)$ & $(-3) 2.15532$ & $(-3) 6.80173$ & $(-2) 1.81749$ \\
\hline 1.8 & $(-6) 1.14908$ & $(-5) 1.48441$ & $(-5) 8.88539$ & $(-4) 1.92238$ & $(-4) 5.34887$ & $(-3) 1.16657$ & $(-3) 2.57418$ & $(-3) 7.68945$ & $(-2) 1$ \\
\hline 1.9 & $(-6) 2.01110$ & $(-5) 2.27091$ & $(-4) 1.23768$ & $(-4) 2.57244$ & $(-4) 6.79123$ & $(-3) 1.42434$ & $(-3) 3.02499$ & $(-3) 8.60436$ & $(-2)$ \\
\hline 2 & $(-6) 3.33349$ & $(-5) 3.33495$ & $(-4) 1.67071$ & $(-4) 3.34955$ & $(-4) 8.43571$ & $(-3) 1.70832$ & $(-3) 3.50586$ & $(-3) 9.54357$ & $(-2) 2.28400$ \\
\hline 2.2 & $(-6) 8.01279$ & $(-5) 6.50454$ & $(-4) 2.81801$ & $(-4) 5.31005$ & $(-3) 1.23328$ & $(-3) 2.35172$ & $(-3) 4.55055$ & $(-2) 1.14851$ & $(-2) 2.59562$ \\
\hline 2.5 & $(-5) 2.31525$ & $(-4) 1.46315$ & $(-4) 5.32875$ & $(-4) 9.32569$ & $(-3) 1.96696$ & $(-3) 3.49204$ & $(-3) 6.30040$ & $(-2) 1.45262$ & $(-2) 3.06236$ \\
\hline 3 & $(-5) 8.61968$ & $(-4) 4$ & $(-3) 1.18485$ & $(-3) 1.89636$ & 56072 & 79912 & $(-3) 9$ & $9(-2)]$ & 33554 \\
\hline 3.5 & $(-4) 2.24269$ & $(-4) 8.41438$ & $(-3) 2.13974$ & $(-3) 3.21572$ & $(-3) 5.56318$ & $(-3) 8.52513$ & $(-2) 1.33076$ & $(-2) 2.53708$ & $3(-2$ \\
\hline 4 & $(-4) 4.65831$ & $(-3) 1.48769$ & $(-3) 3.38798$ & $(-3) 4.85949$ & 91098 & $(-2) 1$ & $(-2) 1.72880$ & $(-2) 3.10313$ & 35521 \\
\hline 4.5 & $(-4)$ & $(-3) 2.34625$ & $(-3) 4.90803$ & $3(-3) 6$ & .05483 & $(-2) 1.49135$ & .14860 & $(-2) 3.67758$ & 09969 \\
\hline 5 & $(-3) 1.33564$ & $(-3) 3.41307$ & $(-3) 6.67426$ & $(-3) 8.97471$ & $(-2) 1.34282$ & $(-2) 1.84582$ & $(-2) 2.58513$ & $(-2) 4.25707$ & $(-2) 6.83350$ \\
\hline 6 & $(-3) 2.77700$ & $(-3) 6.12961$ & $(-2) 1.08437$ & $(-2) 1.39728$ & 97672 & 430 & $(-2)$ & 2216 & 902 \\
\hline 7 & $(-3) 4.79034$ & $(-3) 9.53506$ & $(-2) 1.57118$ & $3(-2) 1.96401$ & $(-2) 2.66907$ & $(-2) 3.41032$ & $(-2) 4.43450$ & $(-2) 6.58571$ & $(-2) 9.66238$ \\
\hline 8 & $(-3) 7.3$ & 35219 & $(-2) 2.11288$ & $(-2) 2.58150$ & $(-2) 3.40329$ & $(-2) 4$ & $(-2) 5.39280$ & $(-2) 7.74031$ & $(-1) 1,10149$ \\
\hline 9 & $(-2)$ & 79931 & $(-2) 2.69764$ & $(-2) 3.23758$ & $(-2) 4.16747$ & $(-2) 5.10682$ & 35999 & $(-2) 8.88144$ & $(-1) 1.23280$ \\
\hline 10 & 078 & 8653 & $(-2) 3.31618$ & $3(-2)$ & .95285 & $(-2) 5.97855$ & $(-2) 7.32979$ & $(-1) 1.00062$ & 6033 \\
\hline 12 & $(-2) 2.17549$ & 439 & $(-2) 4.6$ & $1-2$ & $(-2)$ & 3944 & 26041 & 22001 & 0463 \\
\hline 15 & $(-2) 3.56489$ & $(-2) 5,11488$ & $(-2) 6.70193$ & $(-2) 7.58234$ & $(-2) 9.01152$ & $(-1) 1.03730$ & $(-1) 1.20962$ & 3408 & 4631 \\
\hline 20 & $(-2) 6,19623$ & 25829 & $(-1) 1.02589$ & $(-1) 1.13339$ & $(-1) 1.30377$ & $(-1) 1.46219$ & $(-1) 1.65828$ & $(-1) 2.01703$ & $3(-1) 2$ \\
\hline 24 & $(-2) 8.43927$ & $(-1) 1.08265$ & $(-1) 1 \cdot 30784$ & $(-1) 1.42681$ & $(-1) 1.61299$ & $(-1) 1.78383$ & $(-1) 1.99277$ & $(-1) 2.36895$ & $5(-1) 2.82285$ \\
\hline 30 & $(-1) 1.18587$ & $(-1) 1.46215$ & $(-1)$ & $(-1) 1.84648$ & .04917 & $(-1) 2.23257$ & $(-1) 2.45390$ & $(-1) 2.84554$ & $(-1) 3.30844$ \\
\hline 40 & $(-1$ & 740 & $1-$ & $(-1$ & $1-$ & 9362 & 12428 & 2450 & $\{(-1)$ \\
\hline 60 & $(-1) 2.72306$ & $(-1) 3.07478$ & $(-1) 3.37591$ & $(-1) 3.52577$ & $(-1) 3.74983$ & $(-1) 3.94584$ & $(-1) 4.17502$ & $(-1) 4.56364$ & $(-1) 5.00000$ \\
\hline 120 & $(-1) 4.72805$ & $(-1) 5.06303$ & $(-1) 5.33781$ & $(-1) 5.47092$ & $(-1) 5.66582$ & $(-1) 5.83258$ & $(-1) 6.02348$ & $(-1) 6.33805$ & $(-1) 6.67906$ \\
\hline$\infty$ & 1.00000 & 1.00000 & 1.00000 & 1.00000 & 1.00000 & 1.00000 & 1.00000 & 1.00000 & 1.00000 \\
\hline
\end{tabular}




\begin{tabular}{|c|c|c|c|c|c|c|c|c|c|}
\hline$\nu_{2}$ & $p=0.0001$ & $p=0.001$ & $p=0.005$ & $p=0.01$ & $p=0.025$ & $p=0.05$ & $p=0.1$ & $p=0.25$ & $p=0.5$ \\
\hline 1 & $(-10)$ & $(-8) 1$ & $(-7) 3.28620$ & $(-6) 1.31453$ & $(-6) 8.21805$ & $(-5) 3.29041$ & $(-4) 1.32130$ & $(-4) 8.49264$ & $(-3) 3.79972$ \\
\hline 1.1 & $(-10) 7$ & $(-8) 4.74143$ & $(-7) 8.84663$ & $(-6) 3.11991$ & $(-5) 1.65155$ & $(-5) 5.83323$ & $(-4) 2.06865$ & $(-3) 1.13340$ & $(-3) 4.52397$ \\
\hline 1.2 & $(-9) 2$ & $(-7) 1.38612$ & $(-6) 2.02666$ & $(-6) 6.43530$ & $(-5) 2.96602$ & $(-5) 9.43901$ & $(-4) 3.01966$ & $(-3) 1.44997$ & 26534 \\
\hline 1.3 & $(-9) 9$ & $(-7) 3$ & $(-6) 4.10036$ & $(-5) 1.19143$ & $(-5) 4.88490$ & $(-4) 1.42374$ & $(-4) 4.17649$ & $(-3) 1.79521$ & 01977 \\
\hline 1.4 & $(-8) 2$. & $(-7) 7.54726$ & $(-6) 7.52350$ & $(-5) 2.02607$ & $(-5) 7.51566$ & $(-4) 2.03206$ & $(-4) 5.53653$ & $(-3) 2.16581$ & $(-3) 6.78425$ \\
\hline 1.5 & $(-8) 6.92702$ & $(-6) 1.49245$ & $(-5) 1.27651$ & $(-5) 3.21872$ & $(-4) 1.09497$ & $(-4) 2.77478$ & $(-4) 7.09398$ & $(-3) 2.55884$ & $(-3) 7.55651$ \\
\hline 1.6 & $(-7) 1.52751$ & $(-6) 2.71657$ & $(-5) 2.03228$ & $(-5) 4.83805$ & $(-4) 1.52608$ & $(-4) 3.65500$ & $(-4) 8.84110$ & $(-3) 2.97178$ & $(-3) 8.33483$ \\
\hline 1.7 & $(-7) 3$ & $(-6) 4.61816$ & $(-5) 3.07007$ & $(-5) 6.94765$ & $(-4) 2.05058$ & $(-4) 4.67359$ & $(-3) 1.07691$ & $(-3) 3.40245$ & $(-3) 9.11788$ \\
\hline 1.8 & $(-7) 5$ & $(-6)$ & $(-5) 4.43912$ & 60440 & $(-4) 2.67258$ & $(-4) 5.82972$ & $(-3) 1.28685$ & $(-3) 3.84897$ & 90461 \\
\hline 1.9 & $(-6) 1$ & 13499 & $(-5) 6.18605$ & $(-4) 1.28577$ & $(-4) 3.39479$ & $(-4) 7.12128$ & $(-3) 1.51301$ & $(-3) 4.30970$ & 942 \\
\hline 2 & $(-6) 1$ & $(-5) 1.66749$ & $(-5) 8.35389$ & $(-4) 1.67492$ & $(-4) 4.21874$ & $(-4) 8.54523$ & $(-3) 1.75447$ & $(-3) 4.78322$ & 4860 \\
\hline 2.2 & $(-6) 4.00971$ & $(-5) 3.25501$ & $(-4) 1.41027$ & $(-4)^{2} .65757$ & $(-4) 6.17337$ & $(-3) 1.17752$ & $(-3) 2.27975$ & $(-3) 5.76389$ & $(-2) 1.30741$ \\
\hline 2.5 & $(-5) 1.16002$ & $(-5) 7$ & $(-4$ & $(-4) 4$ & $(-4)$ & $(-3) 1.75114$ & 166 & .30460 & $(-2) 1.54623$ \\
\hline 3 & $(-5) 4.32763$ & $(-4$ & 033 & 2521 & 78926 & $(-3)$ & 33792 & 00122 & 435 \\
\hline 3.5 & 4) 1.12830 & $(-4)$ & $(-3) 1.07701$ & $(-3) 1.61902$ & 80254 & & & 28445 & 34126 \\
\hline 4 & $(-4) 2 \cdot 34844$ & $(-4) 7$ & $(-3) 1.70925$ & $(-3) 2.45253$ & $(-3) 3$ & $(-3)=$ & $(-3) 8.75210$ & $(-2) 1.57640$ & $(-2) 2.73615$ \\
\hline 4.5 & $(-4) 4.20271$ & $(-3) 1$ & $(-3) 2.48198$ & $(-3) 3.43582$ & $(-3) 5.34170$ & $(-3) 7.56048$ & $(-2) 1.09103$ & $(-2) 1.87463$ & $(-2) 3.12860$ \\
\hline 5 & $(-4) 6.76169$ & $(-3) 1.72875$ & $(-3) 3.38330$ & $(-3) 4.55202$ & $(-3) 6.81835$ & $(-3) 9.38413$ & $(-2) 1.31671$ & $(-2) 2.17744$ & $(-2) 3.51841$ \\
\hline 6 & $(-3) 1.41185$ & $(-3) 3.11892$ & $(-3)$ & $(-3) 7.12350$ & $(-2) 1$ & 33168 & 79064 & .79228 & 28964 \\
\hline 7 & $(-3) 2.44603$ & $(-3) 4.87440$ & $(-3) 8.04415$ & $(-2) 1.00651$ & $(-2) 1.37022$ & $(-2) 1.75398$ & $(-2) 2.28657$ & 41434 & 941 \\
\hline 8 & $(-3) 3.76261$ & $(-3) 6$ & $(-2) 1.08727$ & $(-2) 1.32995$ & $(-2) 1.75688$ & $(-2) 2.19762$ & 79777 & $(-2) 4.03964$ & $(-2)$ \\
\hline 9 & $(-3) 5.33758$ & $(-3) 9.2863 \mathrm{I}$ & $(-2) 1.39532$ & $(-2) 1.67681$ & $(-2) 2.16338$ & $(-2) 2.65720$ & $(-2) 3.31966$ & $(-2) 4.66558$ & $(-2) 6.53455$ \\
\hline 10 & $(-3) 7.14507$ & $(-2) 1$ & $(-2) 1.7$ & 1258 & $(-2) 2.58538$ & $(-2) 3.12877$ & 4894 & $(-2) 5.29036$ & $(-2) 7.26017$ \\
\hline 12 & 596 & $(-2) 1.75652$ & $(-2) 2.43016$ & $(-2) 2.81735$ & $(-2) 3.46337$ & $(-2) 4.09669$ & 059 & $(-2) 6.53169$ & $(-2) 8.67850$ \\
\hline 15 & $(-2) 1.88693$ & $(-2) 2.71742$ & $(-2) 3.57431$ & $(-2) 4.05258$ & $(-2) 4.83349$ & 58271 & 53861 & 36238 & $(-1)$ \\
\hline 20 & $(-2) 3.35364$ & 9174 & 0701 & $(-2)$ & $(-2) 7$ & $(-2) \varepsilon$ & 20850 & 13065 & $(-1)$ \\
\hline 24 & $(-2) 4.64714$ & $(-2) 5.99568$ & $(-2) 7.28247$ & $(-2) 7.96827$ & $(-2) 9.04999$ & $(-1) 1.00521$ & $(-1) 1.12901$ & $(-1) 1.35560$ & $(-1) 1.63572$ \\
\hline 30 & $(-2) 6.69313$ & $(-2) 8.30695$ & $(-2) 9.80479$ & $(-1) 1.05898$ & $(-1) 1.18124$ & $(-1) 1.29302$ & $(-1) 1.42946$ & $(-1) 1.67518$ & $(-1) 1.97324$ \\
\hline 40 & $(-1) 1.01992$ & $(-1) 1$ & $(-1)$ & $(-1)$ & $(-1)$ & 4525 & 600 & 6261 & $(-1)$ \\
\hline 60 & $(-1) 1.70038$ & $(-1) 1.93641$ & $(-1) 2.14214$ & $(-1) 2.24586$ & $(-1) 2.40265$ & $(-1) 2.54160$ & $(-1) 2.70626$ & $(-1) 2.99131$ & $(-1)$ \\
\hline 120 & $(-1) 3.34705$ & $(-1) 3.61436$ & $(-1) 3.83802$ & $(-1) 3.94790$ & $(-1) 4.11070$ & $(-1) 4.25191$ & $(-1) 4.4$ & $(-1) 4.69179$ & $(-1) 5.00000$ \\
\hline$\infty$ & 1.00000 & 1.00000 & 1.00000 & 1.00000 & 1.00000 & 1.00000 & 1.00000 & 1.00000 & 1.00000 \\
\hline
\end{tabular}


TABLE III

VALUES OF THE COMPLETE BETA FUNC TION, $\mathrm{B}\left(v_{2} / 2, v_{1} / 2\right)$. 
TABLE III. Values of the Complete Beta Function, $B\left(v_{2} / 2, v_{1} / 2\right)$.

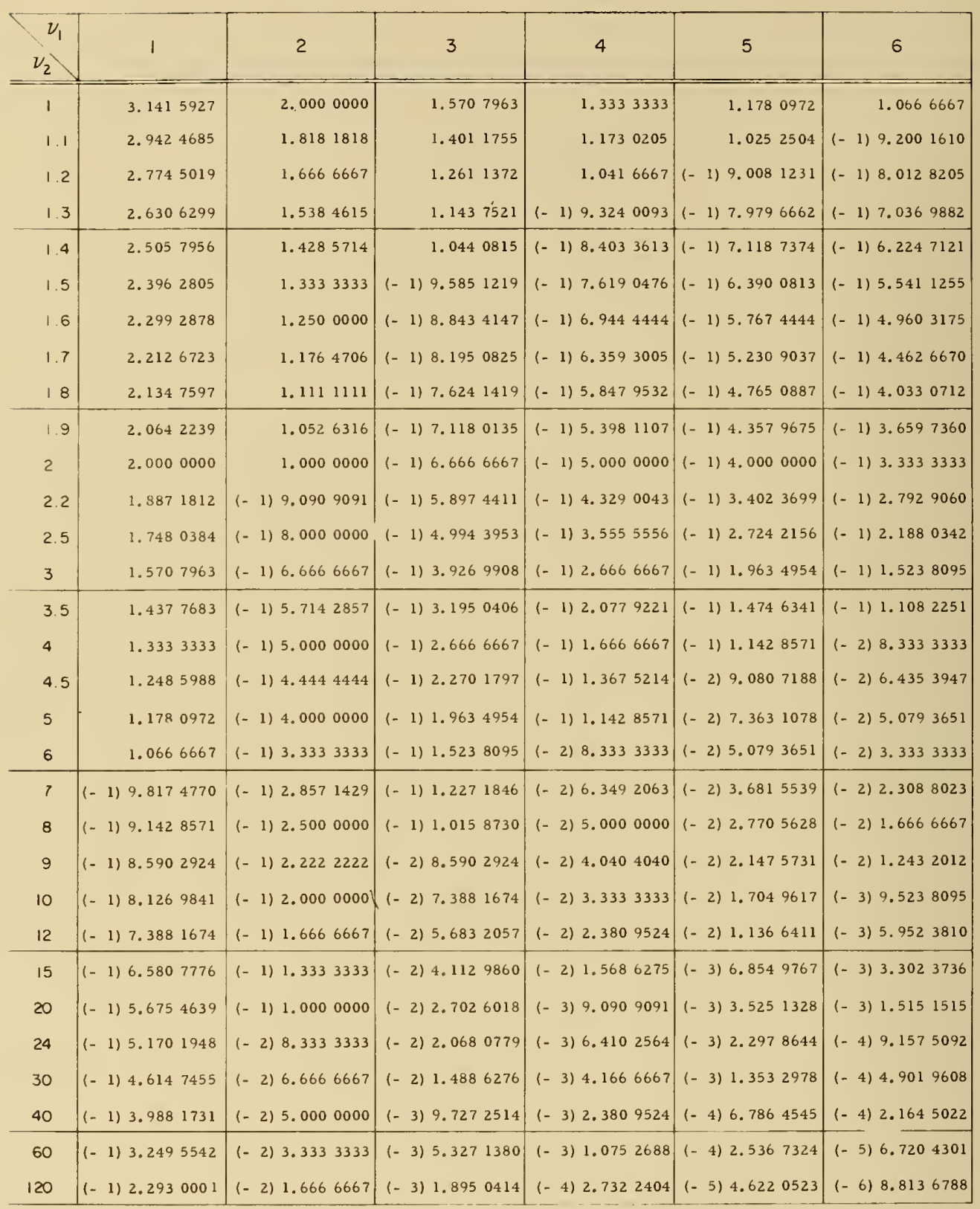

he numbers in parentheses indicate the power of ten by which the number following is to be multiplied, e.g., $(-1) 1.2345678=0.12345678$. 
TABLE III. Values of the Complete Beta Function, B $\left(v_{2} / 2, v_{1} / 2\right)$.

\begin{tabular}{|c|c|c|c|c|c|c|}
\hline$v_{1}$ & 7 & 8 & 9 & 10 & 12 & 15 \\
\hline 1 & $(-1) 9.8174770$ & $(-1) 9.1428571$ & $(-1) 8.5902924$ & $(-1) 8.1269841$ & $(-1) \quad 7.388 \quad 1674$ & $(-1) 6.5807776$ \\
\hline 1.1 & $(-1) 8.4036915$ & (- 1) 7.7747839 & $(-1) 7.2624495$ & $(-1) 6.8349749$ & (- 1) 6.1576350 & $(-1) 5.4242015$ \\
\hline 1.2 & $(-1) 7.2646154$ & $(-1) 6.6773504$ & $(-1) 6.2015010$ & $(-1) 5.8063917$ & (- 1) 5.1842783 & $(-1) 4.5167600$ \\
\hline 1.3 & $(-1) 6.3330684$ & $(-1) 5.7838259$ & $(-1) 5.3411420$ & $(-1) 4.9753341$ & (- 1) 4.4029505 & $(-1) 3.7943230$ \\
\hline 1.4 & $(-1) 5.5615136$ & $(-1) 5.0470639$ & $(-1) 4.6345947$ & $(-1) 4.2953735$ & $\{-1) 3.7678715$ & $(-1) 3.2119797$ \\
\hline 1.5 & $(-1) 4.9154471$ & $(-1) 4.4329004$ & $(-1) 4.0480153$ & $(-1) 3.7329688$ & $(-1) 3.2460598$ & (- 1) 2.7374953 \\
\hline 1.6 & $(-1) 4.3692760$ & $(-1) 3.9160401$ & (- 1) 3.5563875 & (- 1) 3.2633668 & $(-1) 2.8132472$ & $(-1) 2.3472446$ \\
\hline 1.7 & $(-1) 3.9036595$ & $(-1) 3.4774029$ & $(-1) 3.1408754$ & $(-1) 2.8679611$ & (- 1) 2.4512488 & $(-1) 2.0235989$ \\
\hline 1.8 & (- 1) 3.5037417 & $(-1) 3.1023624$ & $(-1) 2.7870672$ & (- 1) 2.5325408 & (- 1) 2.1462210 & $(-1) \quad 1.7531963$ \\
\hline 1.9 & $(-1) 3.1579474$ & $(-1) 2.7795464$ & $(-1) 2.4837789$ & (- 1) 2.2460981 & $\{-1) 1.8874774$ & $(-1) \quad 1.5257697$ \\
\hline 2 & $(-1) 2.8571429$ & $(-1) 2.5000000$ & $(-1) 2.2222222$ & $(-1) 2.0000000$ & $(-1) 1.6666667$ & $(-1) 1.3333333$ \\
\hline 2. 2 & $(-1) 2.3627569$ & $(-1) 2.0435898$ & (- 1) 1.7977498 & $(-1) 1.6028155$ & $(-1) 1.3137832$ & $(-1) 1.0296088$ \\
\hline 2.5 & $(-1) \quad 1.816 \quad 1438$ & (- 1) 1.5444947 & $(-1) 1.3382112$ & $(-1) 1.1767579$ & $(-2) 9.4140631$ & $(-2) 7.1571463$ \\
\hline 3 & $(-1) 1.2271846$ & $(-1) 1.0158730$ & $(-2) 8$ & $(-2) \quad 7.388 \quad 1674$ & $(-2) 5.6832057$ & $(-2) 4.1129860$ \\
\hline 3.5 & $(-2) 8.674 \quad 3184$ & $(-2) 6.9993165$ & $(-2) 5$. & $(-2) 4.8690897$ & $(-2) 3.6067331$ & $(-2) 2.4886320$ \\
\hline 4 & $(-2) 6.3492063$ & $(-2) 5.0000000$ & $(-2) 4.0404040$ & $(-2) 3.3333333$ & $(-2) 2.3809524$ & $(-2) 1.5686275$ \\
\hline 4.5 & $(-2) 4.7793257$ & $(-2) 3.6773684$ & $(-2) 2.9091548$ & $(-2) 2.3535158$ & $(-2) 1.6231143$ & $(-2) 1.0224495$ \\
\hline 5 & $(-2) 3.6815539$ & $(-2) 2.7705628$ & $(-2) 2.1475731$ & $(-2) 1.7049617$ & $(-2) 1.1366411$ & $(-3) 6.8549767$ \\
\hline 6 & (1- 2) 2.3088023 & $(-2) 1.6666667$ & $(-2) 1.2432012$ & $(-3) 9.5238095$ & $(-3) 5.9523810$ & $(-3) 3.3023736$ \\
\hline 7 & $1-2) 1.5339808$ & $(-2) 1.0656011$ & $(-3) 7.6699039$ & $(-3) 5.6832057$ & $(-3) 3.3430622$ & $(-3) 1.713$ \\
\hline 8 & $(-2) 1.0656011$ & $(-3) 7.1428571$ & $(-3) 4.9728050$ & $(-3) 3.5714286$ & $(-3) 1.9841270$ & $(-4) 9.4353531$ \\
\hline 9 & $(-3) 7.6699039$ & $(-3) 4.9728050$ & $(-3) 3.3555830$ & $(-3) 2.3401435$ & $(-3) 1.2316545$ & $(-4) 5.4528223$ \\
\hline 10 & $(-3) 5.6832057$ & $(-3) 3.5714286$ & (- 3) 2.3401435 & $(-3) 1.5873016$ & $(-4) 7.9365079$ & $(-4) 3.2818619$ \\
\hline 12 & $(-3) 3.3430622$ & $(-3) \quad 1.984 \quad 1270$ & $(-3) 1.2316545$ & $(-4) 7.9365079$ & $(-4) 3.6075036$ & $(-4) 1.3127448$ \\
\hline 15 & $(-3) 1.7137442$ & $(-4) 9.4353531$ & $(-4) 5$ & $(-4) 3.2818619$ & $(-4) 1.3127448$ & $(-5) 4.0165879$ \\
\hline 20 & (- 4) 7.0502657 & $(-4) 3.4965035$ & $(-4) 1.8278467$ & $(-5) 9.9900100$ & $(-5) 3.3300033$ & $(-6) 7.9294794$ \\
\hline 24 & $(-4) 3.9618351$ & $(-4) 1.8315018$ & $(-5) 8.9460793$ & $(-5) 4.5787546$ & $(-5) 1.3466925$ & $(-6) 2.6941860$ \\
\hline 30 & $(-4) 1.9332826$ & $(-5) 8.1699346$ & $(-5) 3.6575617$ & $(-5) 1.7199862$ & $(-6) 4.2999656$ & $(-7) 6.8462583$ \\
\hline 40 & $(-5) 7.5405050$ & $(-5) 2.823 \quad 2637$ & $(-5) 1.1230539$ & $(-6) 4.705 \quad 4395$ & $(-7) 9.4108790$ & $(-7) 1.0912821$ \\
\hline 60 & $(-5) 1.9513326$ & $(-6) 6.1094819$ & $(-6) 2.0387057$ & $(-7) 7.1876258$ & $(-7) 1.0268037$ & $(-9) 7.3367342$ \\
\hline 120 & $(-6) 1.8488209$ & $(-7) 4.1969899$ & $(-7) 1.0190351$ & $(-8) 2.6231187$ & $(-9) 2.0177836$ & $(-11) 5.8351908$ \\
\hline
\end{tabular}

The numbers in parentheses indicate the power of ten by which the number following is to be multiplied, e.g., $(-1) 1.2345678=0.12345678$. 
TABLE LI. Values of the Complete Beta Function, $B\left(v_{2} / 2, v_{1} / 2\right)$.

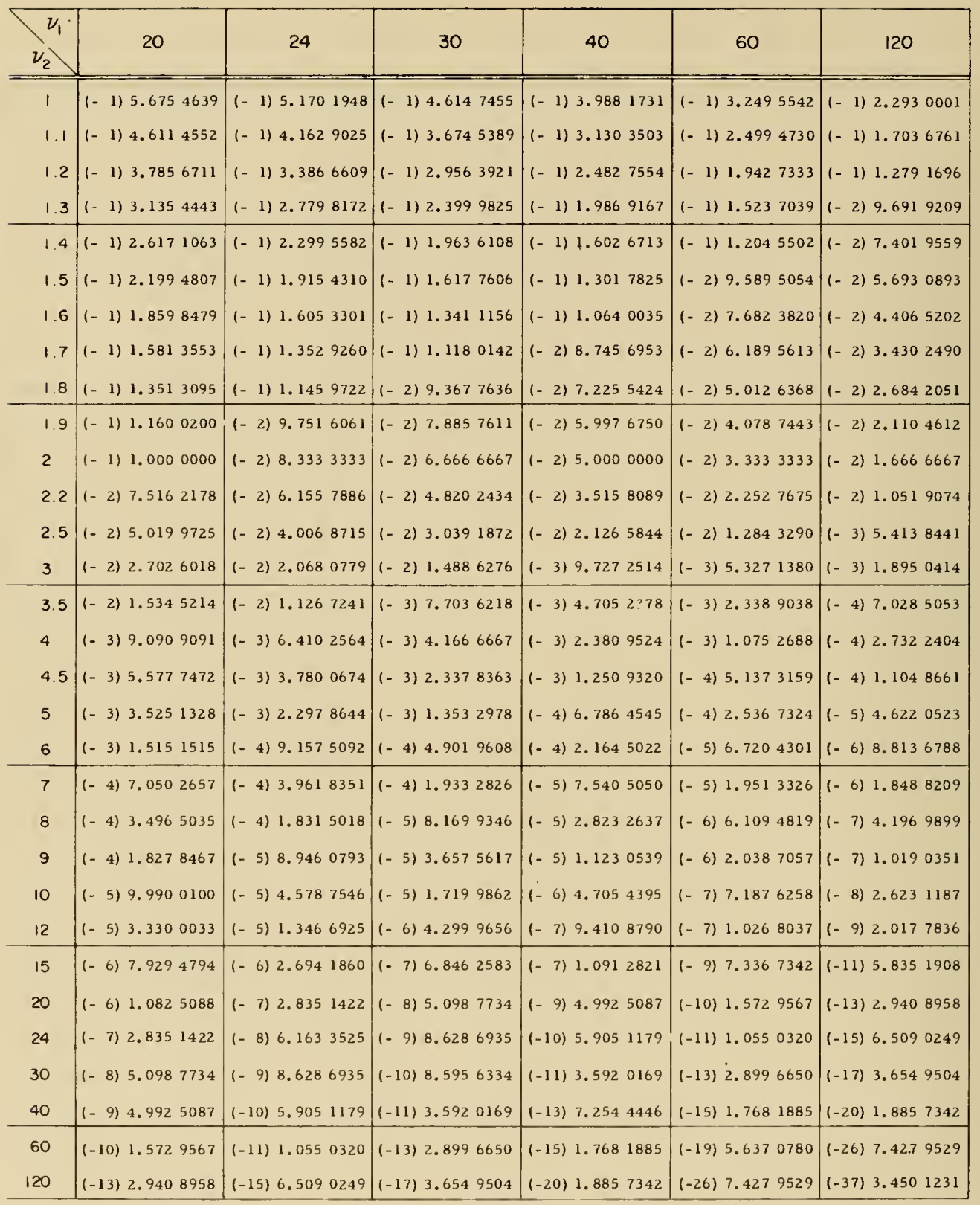

The numbers in parentheses indicate the power of ten by which the number following is to be multiplied, e. $g .,(-1) 1.2345678=0.12345678$. 




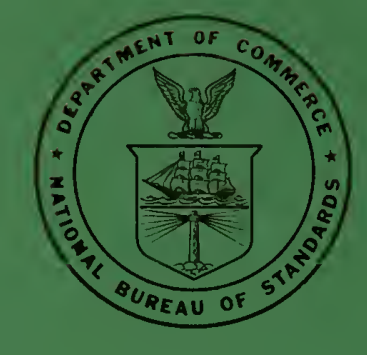

